Supplementary Information for

\title{
Quantum Chemistry-Informed Active Learning to Accelerate the Design and Discovery of Sustainable Energy Storage Materials
}

Hieu A. Doan, ${ }^{\dagger, \ddagger}$ Garvit Agarwal, ${ }^{\dagger, \ddagger}$ Hai Qian, ${ }^{\natural, \S, \ddagger}$ Michael J. Counihan, ${ }^{\natural, \S, \ddagger}$ Joaquín Rodríguez-

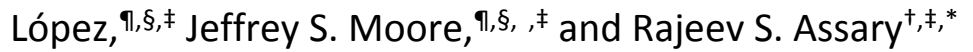

${ }^{\dagger}$ Materials Science Division, Argonne National Laboratory and ${ }^{\ddagger}$ Joint Center for Energy Storage Research, Argonne National Laboratory, Lemont, IL 60439, USA

"The Beckman Institute for Advanced Science and Technology, §Department of Chemistry, and Department of Materials Science and Engineering, University of Illinois at Urbana-Champaign, Urbana, IL 61801, USA 
Table S1 List of 133 identified HBEs with computed oxidation potential ( $\left.E^{\circ}\right)$ in the range of $1.40 \mathrm{~V}$ to $1.70 \mathrm{~V}$

\begin{tabular}{|c|c|c|c|}
\hline HBE \# & SMILES & 2-D Structure & Ex (V vs. NHE) \\
\hline 1 & $\begin{aligned} \mathrm{O}(\mathrm{C}) \mathrm{C} 1=\mathrm{CC}=\mathrm{C}(\mathrm{C}(\mathrm{C} 2 & =\mathrm{CC}=\mathrm{CC}=\mathrm{C} 2)(\mathrm{C} 3=\mathrm{CC}=\mathrm{CC}=\mathrm{C} 3) \mathrm{C}(\mathrm{C} 4= \\
\mathrm{CC} & =\mathrm{CC}=\mathrm{C} 4) \mathrm{OC}) \mathrm{C}=\mathrm{C} 1\end{aligned}$ & & 1.52 \\
\hline 2 & $\mathrm{O}(\mathrm{C}) \mathrm{C} 1=\mathrm{CC}=\mathrm{C}(\mathrm{C}(\mathrm{C} 2=\mathrm{CC}=\mathrm{CC}=\mathrm{C} 2)(\mathrm{C}) \mathrm{C}(\mathrm{C}) \mathrm{OC}) \mathrm{C}=\mathrm{C} 1$ & & 1.52 \\
\hline 3 & $\mathrm{O}(\mathrm{C}) \mathrm{C} 1=\mathrm{CC}=\mathrm{C}(\mathrm{C}(\mathrm{C} 2=\mathrm{CC}=\mathrm{CC}=\mathrm{C} 2)(\mathrm{C}) \mathrm{C}(\mathrm{C}) \mathrm{OCOC}) \mathrm{C}=\mathrm{C} 1$ & & 1.53 \\
\hline 4 & $\begin{aligned} \mathrm{O}(\mathrm{C}) \mathrm{C} 1=\mathrm{CC}=\mathrm{C}(\mathrm{C}(\mathrm{C} 2=\mathrm{CC}=\mathrm{CC}=\mathrm{C} 2)(\mathrm{C} 3=\mathrm{CC}=\mathrm{CC}=\mathrm{C} 3) \mathrm{C}(\mathrm{C}) \mathrm{O} \\
\mathrm{COC}) \mathrm{C}=\mathrm{C} 1\end{aligned}$ & & 1.54 \\
\hline 5 & $\mathrm{O}(\mathrm{C}) \mathrm{C} 1=\mathrm{CC}=\mathrm{C}(\mathrm{C}(\mathrm{C})(\mathrm{C}) \mathrm{C}(\mathrm{C}) \mathrm{OCOC}) \mathrm{C}=\mathrm{C} 1$ & & 1.54 \\
\hline
\end{tabular}




\begin{tabular}{|c|c|c|}
\hline 6 & $\begin{aligned} \mathrm{O}(\mathrm{C}) \mathrm{C} 1=\mathrm{CC}=\mathrm{C}(\mathrm{C}(\mathrm{C} 2=\mathrm{CC}=\mathrm{CC}=\mathrm{C} 2)(\mathrm{C}) \mathrm{C}(\mathrm{C} 3=\mathrm{CC}=\mathrm{CC}=\mathrm{C} 3) \mathrm{O} \\
\mathrm{C}) \mathrm{C}=\mathrm{C} 1\end{aligned}$ & 1.54 \\
\hline 7 & $\begin{aligned} \mathrm{O}(\mathrm{C}) \mathrm{C} 1=\mathrm{CC}=\mathrm{C}(\mathrm{C}(\mathrm{C} 2=\mathrm{CC}=\mathrm{CC}=\mathrm{C} 2)(\mathrm{C}) \mathrm{C}(\mathrm{C}) \mathrm{OC}=\mathrm{CC}=\mathrm{CC}=\mathrm{C} \\
\text { 3) } \mathrm{C}=\mathrm{C} 1\end{aligned}$ & 1.54 \\
\hline 8 & $\mathrm{O}(\mathrm{C}) \mathrm{C} 1=\mathrm{CC}=\mathrm{C}(\mathrm{C}([\mathrm{H}])(\mathrm{C}) \mathrm{C}(\mathrm{C} 2=\mathrm{CC}=\mathrm{CC}=\mathrm{C} 2) \mathrm{OC}) \mathrm{C}=\mathrm{C} 1$ & 1.55 \\
\hline 9 & $\mathrm{O}(\mathrm{C}) \mathrm{C} 1=\mathrm{CC}=\mathrm{C}(\mathrm{C}([\mathrm{H}])(\mathrm{C}) \mathrm{C}(\mathrm{C}) \mathrm{OC} 2=\mathrm{CC}=\mathrm{CC}=\mathrm{C} 2) \mathrm{C}=\mathrm{C} 1$ & 1.55 \\
\hline 10 & $\mathrm{O}(\mathrm{C}) \mathrm{C} 1=\mathrm{CC}=\mathrm{C}(\mathrm{C}(\mathrm{C})(\mathrm{C}) \mathrm{C}(\mathrm{C}) \mathrm{OC}) \mathrm{C}=\mathrm{C} 1$ & 1.55 \\
\hline
\end{tabular}




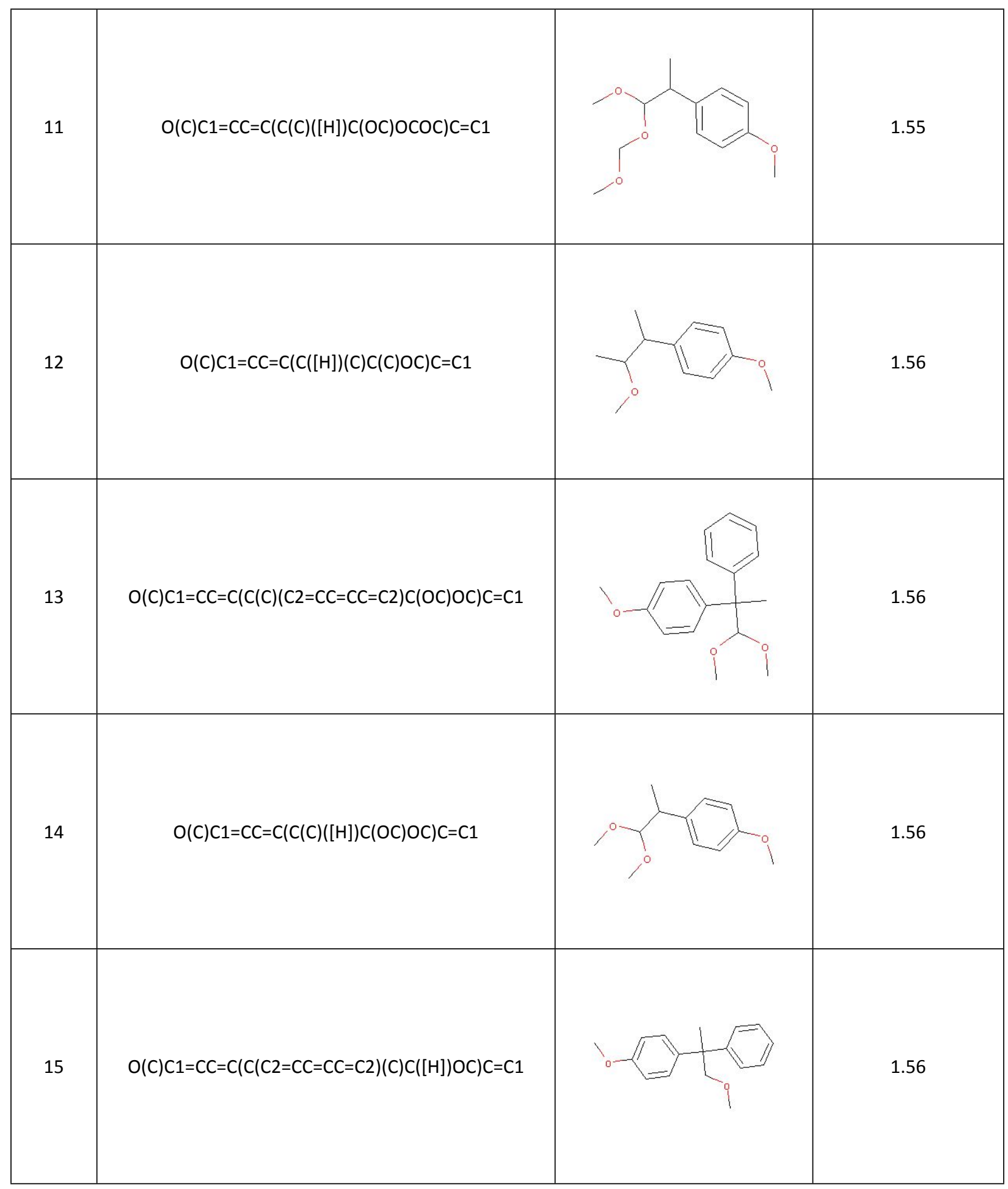




\begin{tabular}{|c|c|c|}
\hline 16 & $\begin{aligned} \mathrm{O}(\mathrm{C}) \mathrm{C} 1=\mathrm{CC}=\mathrm{C}(\mathrm{C}(\mathrm{C})(\mathrm{C}) \mathrm{C}(\mathrm{C} 2=\mathrm{CC}=\mathrm{CC}=\mathrm{C} 2) \mathrm{OC} 3=\mathrm{CC}=\mathrm{CC}=\mathrm{C} \\
\text { 3) } \mathrm{C}=\mathrm{C} 1\end{aligned}$ & 1.56 \\
\hline 17 & $\begin{array}{c}\mathrm{O}(\mathrm{C}) \mathrm{C} 1=\mathrm{CC}=\mathrm{C}(\mathrm{C}(\mathrm{C})(\mathrm{C} 2=\mathrm{CC}=\mathrm{CC}=\mathrm{C} 2) \mathrm{C}(\mathrm{C} 3=\mathrm{CC}=\mathrm{CC}=\mathrm{C} 3) \mathrm{O} \\
\mathrm{C} 4=\mathrm{CC}=\mathrm{CC}=\mathrm{C} 4) \mathrm{C}=\mathrm{C} 1\end{array}$ & 1.57 \\
\hline 18 & $\mathrm{O}(\mathrm{C}) \mathrm{C} 1=\mathrm{CC}=\mathrm{C}(\mathrm{C}([\mathrm{H}])([\mathrm{H}]) \mathrm{C}(\mathrm{C}) \mathrm{OC} 2=\mathrm{CC}=\mathrm{CC}=\mathrm{C} 2) \mathrm{C}=\mathrm{C} 1$ & 1.57 \\
\hline 19 & $\begin{aligned} \mathrm{O}(\mathrm{C}) \mathrm{C} 1=\mathrm{CC}=\mathrm{C}(\mathrm{C}(\mathrm{C} 2=\mathrm{CC}=\mathrm{CC}=\mathrm{C} 2)(\mathrm{C}) \mathrm{C}(\mathrm{OC}) \mathrm{OC} 3=\mathrm{CC}=\mathrm{CC}= \\
\mathrm{C} 3) \mathrm{C}=\mathrm{C} 1\end{aligned}$ & 1.57 \\
\hline 20 & $\begin{array}{c}\mathrm{O}(\mathrm{C}) \mathrm{C} 1=\mathrm{CC}=\mathrm{C}(\mathrm{C}([\mathrm{H}])(\mathrm{C}) \mathrm{C}(\mathrm{C} 2=\mathrm{CC}=\mathrm{CC}=\mathrm{C} 2) \mathrm{OC} 3=\mathrm{CC}=\mathrm{CC}= \\
\mathrm{C} 3) \mathrm{C}=\mathrm{C} 1\end{array}$ & 1.57 \\
\hline
\end{tabular}




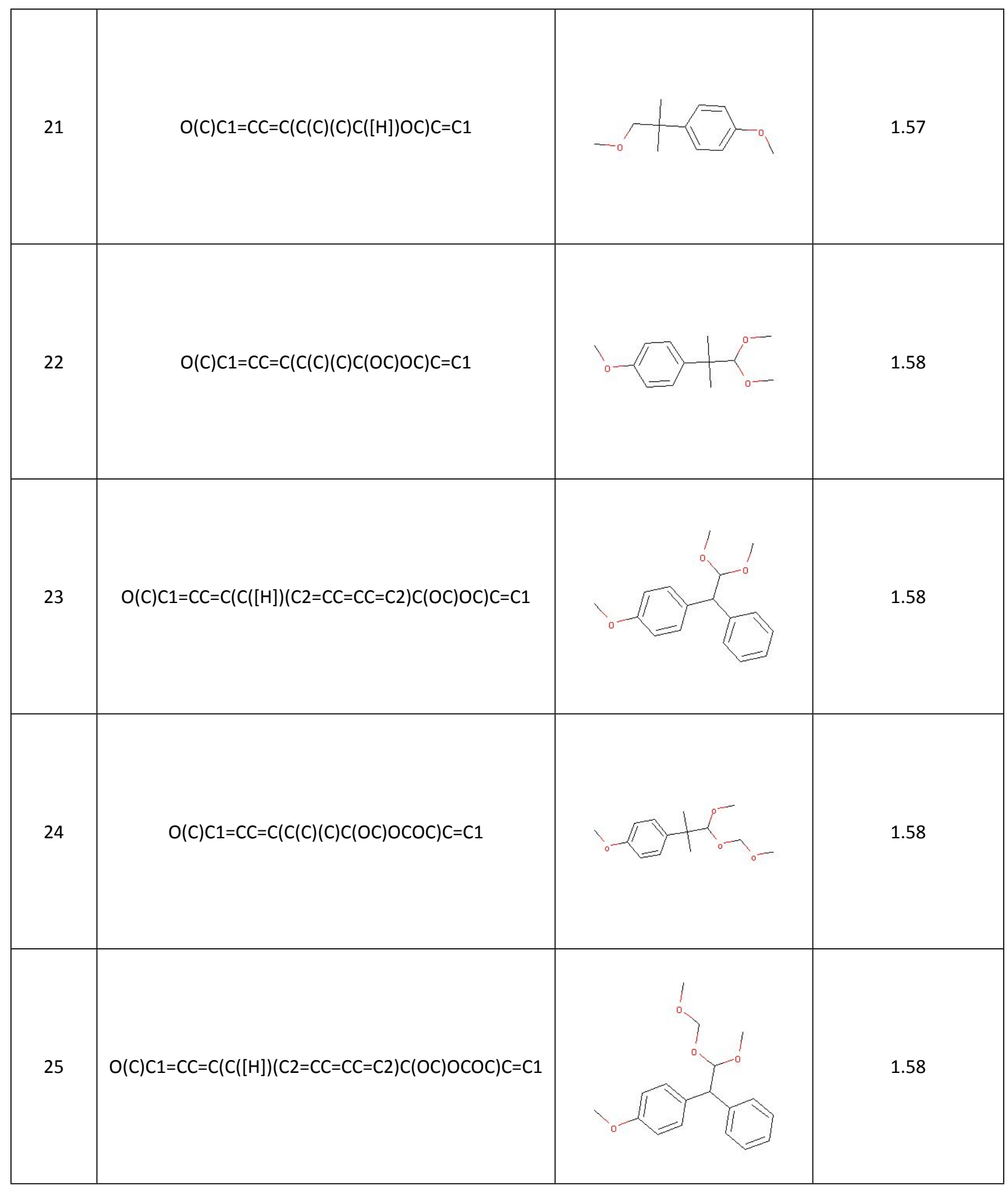




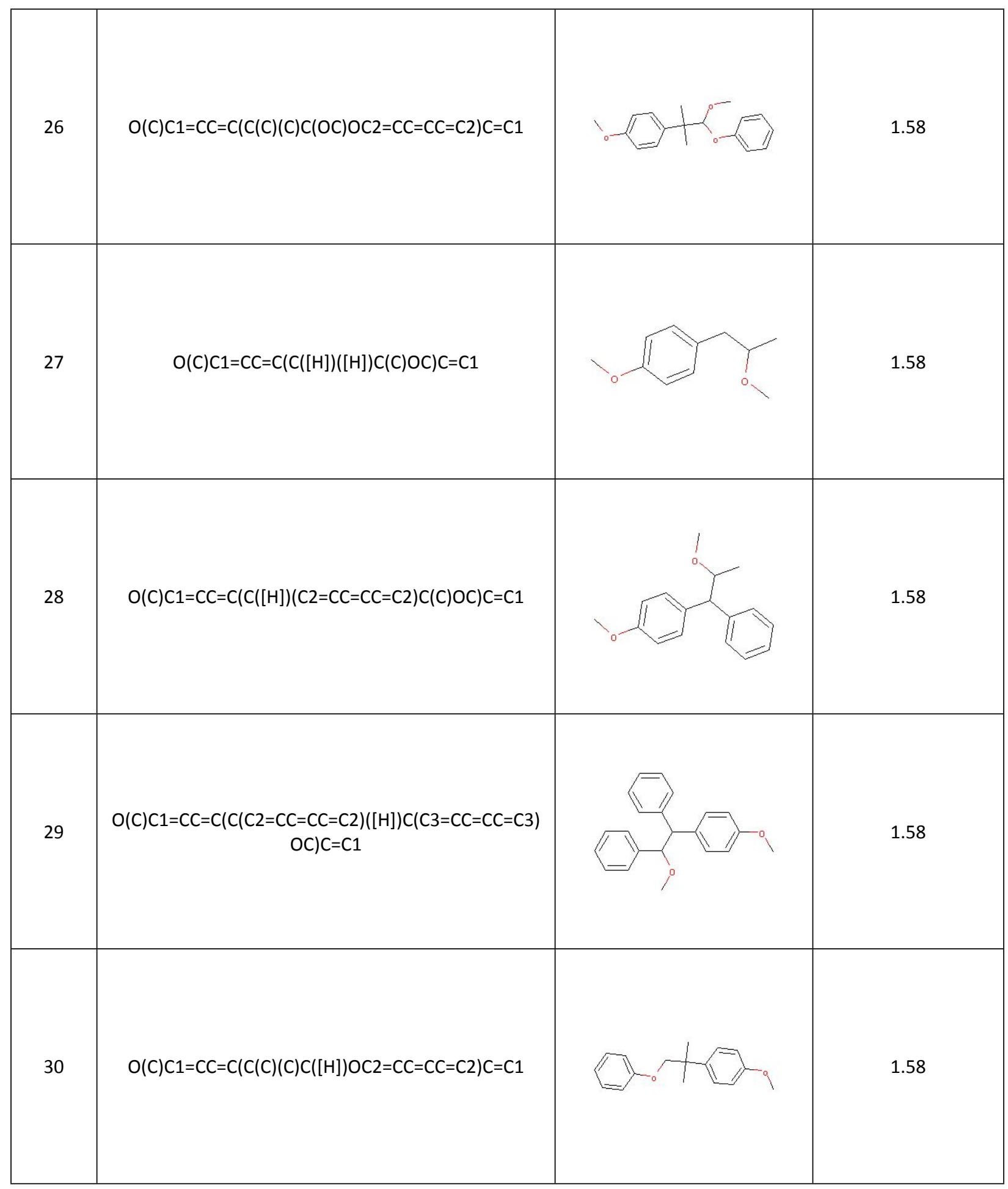




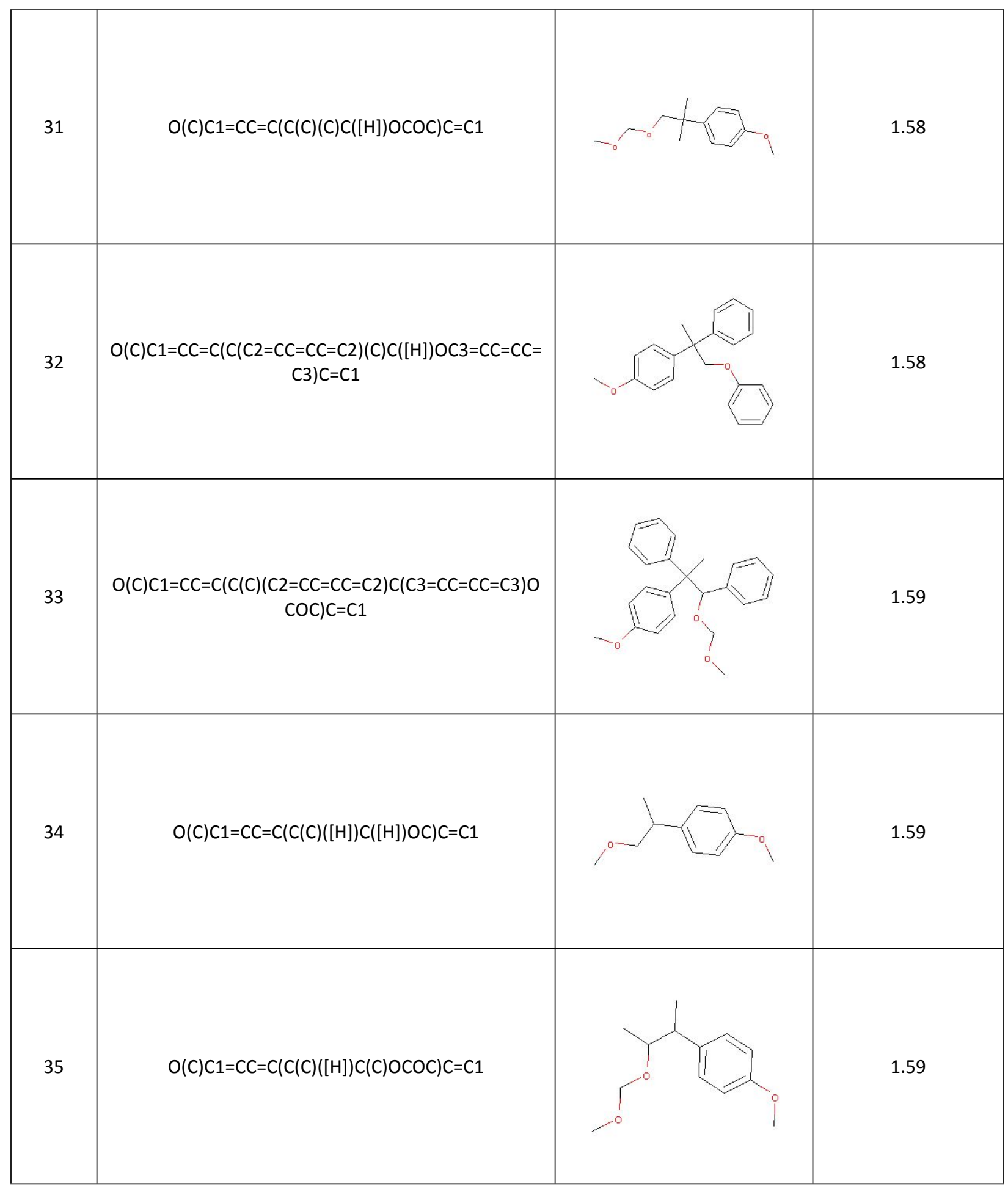




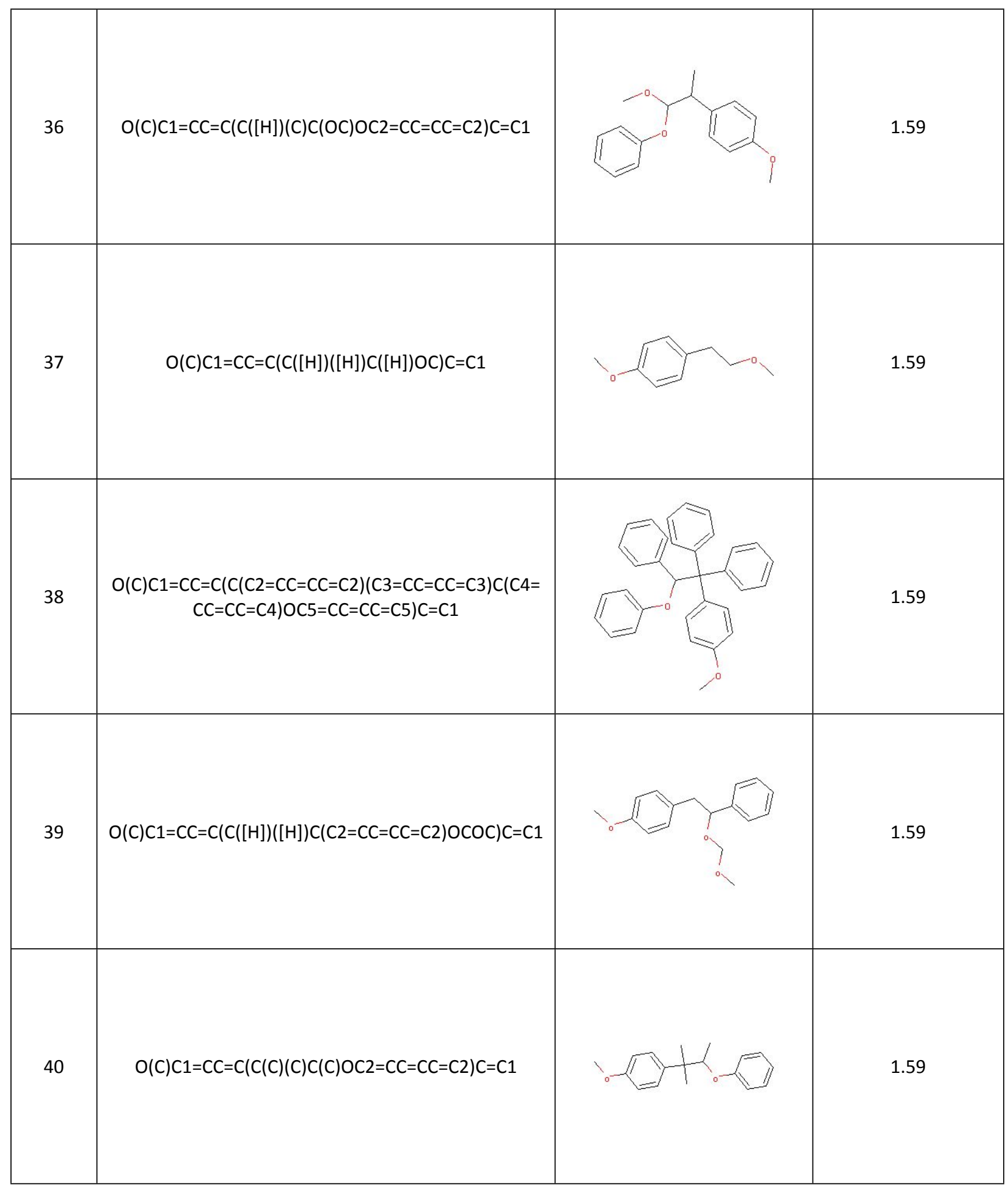




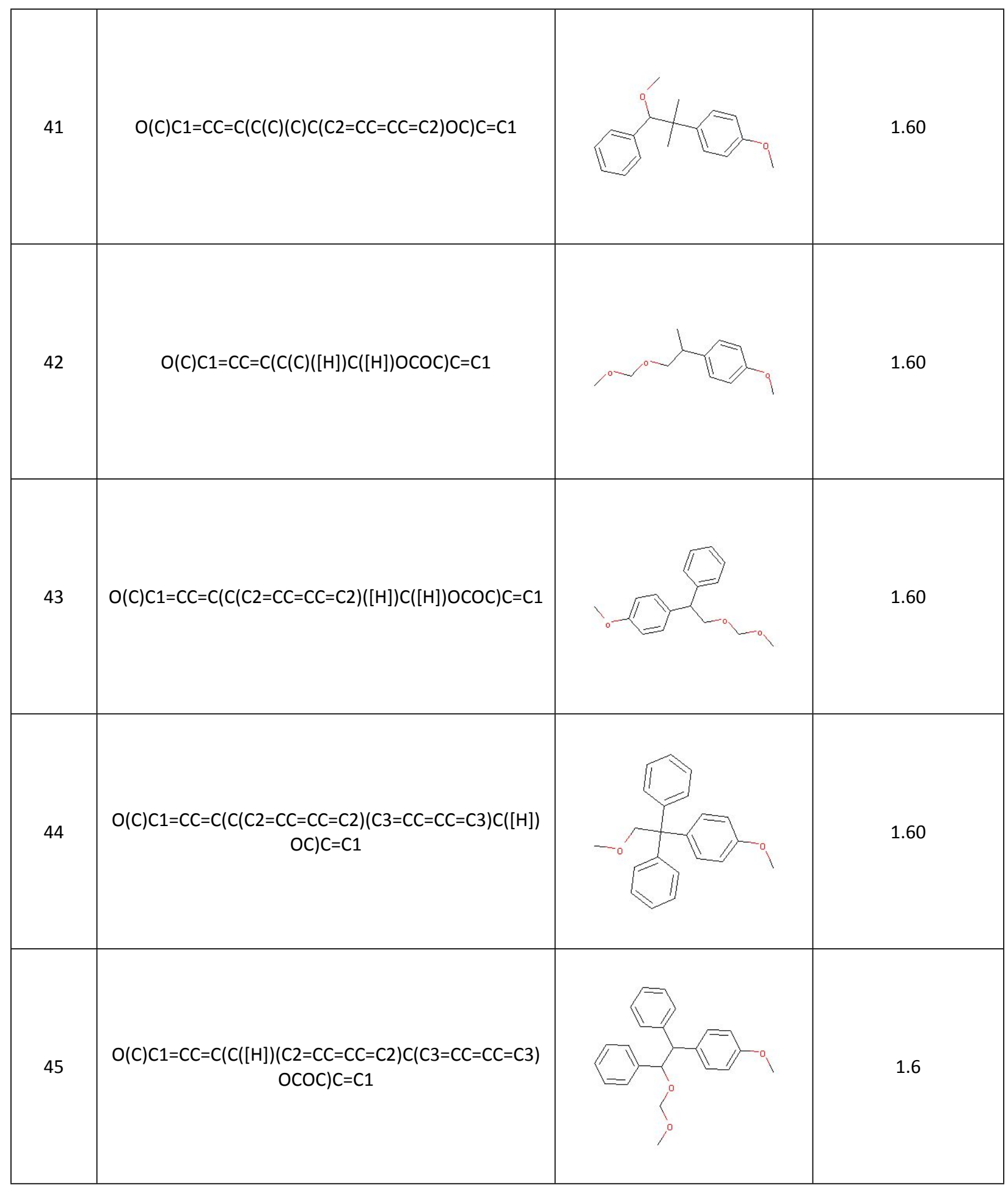




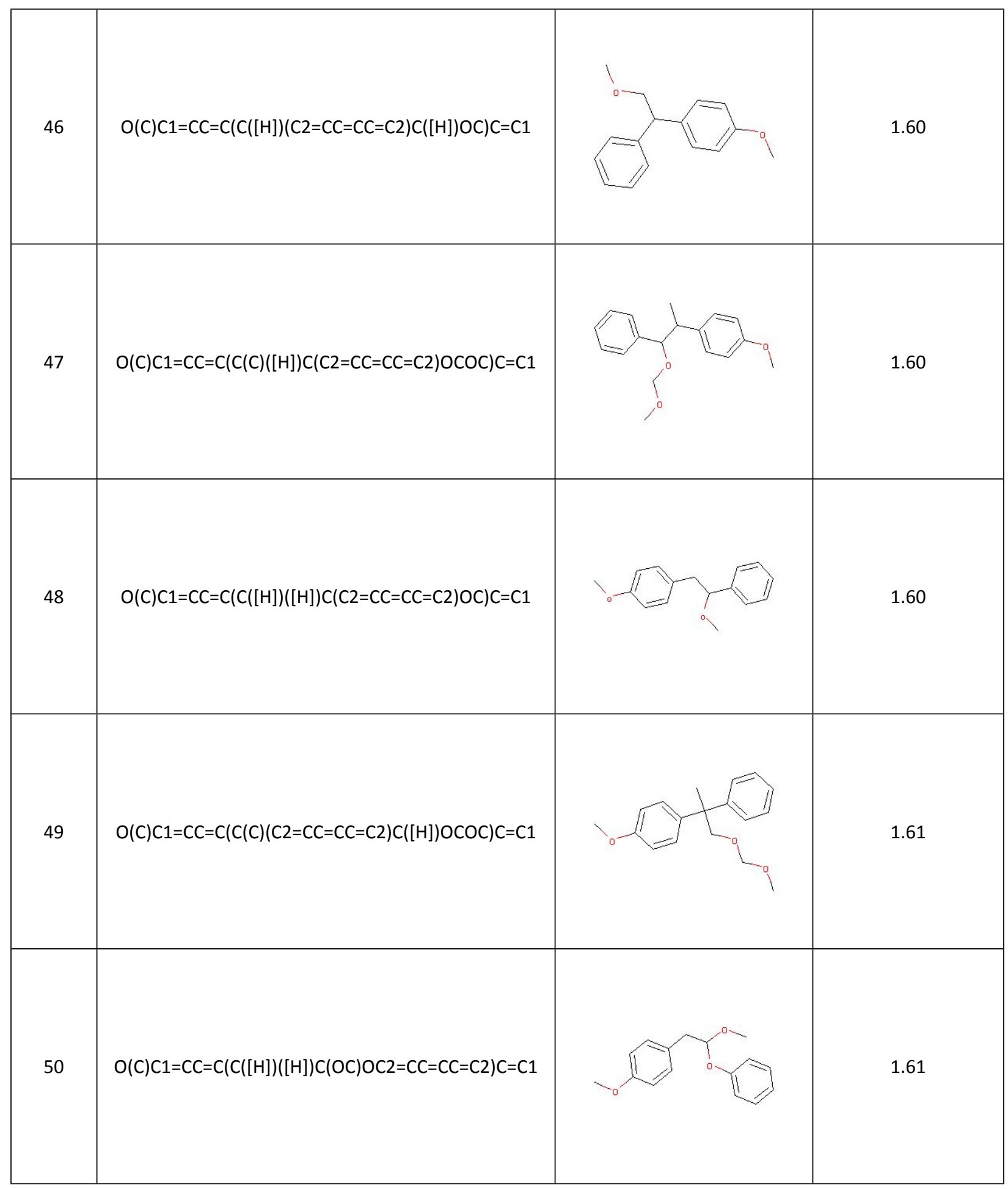




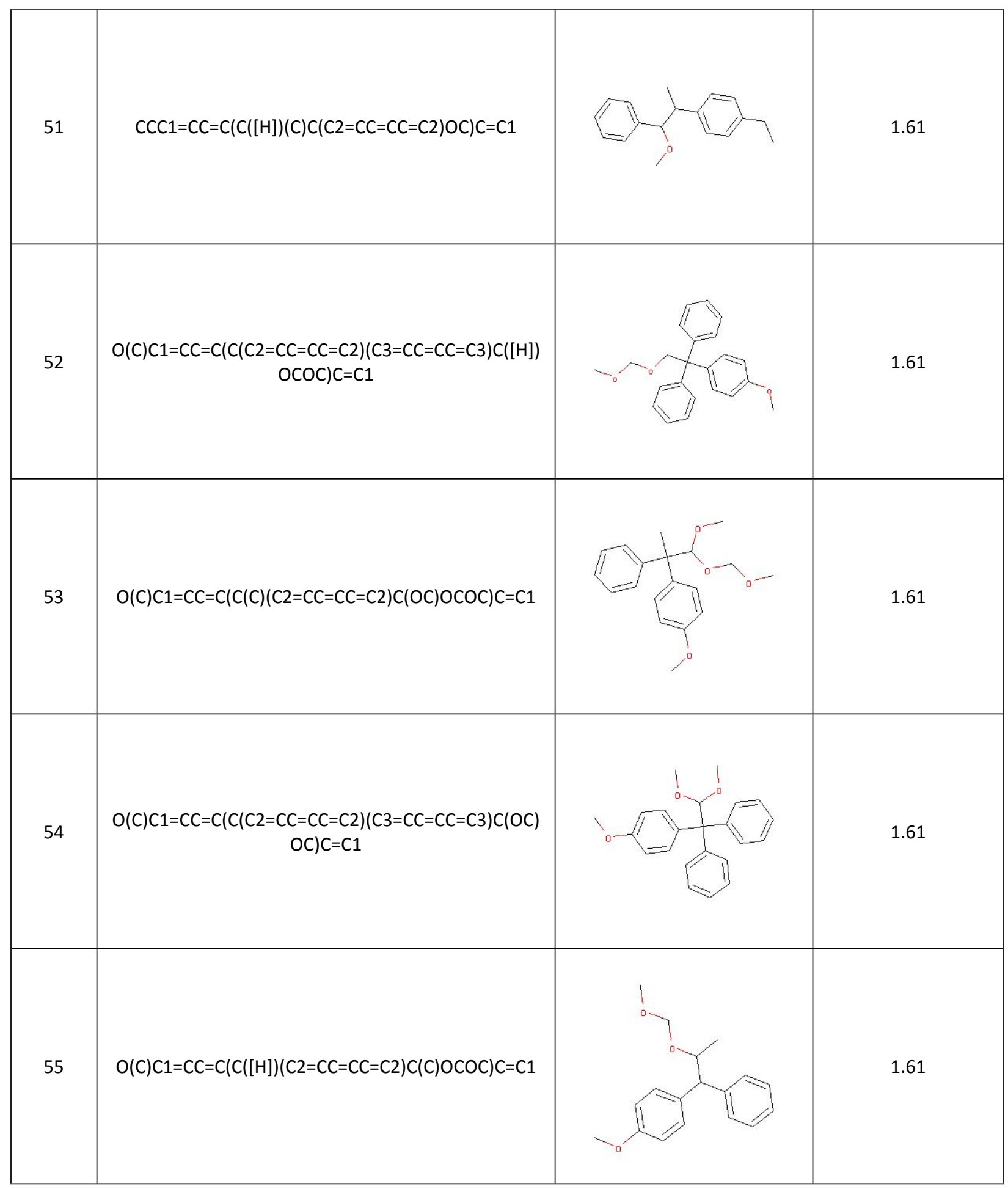




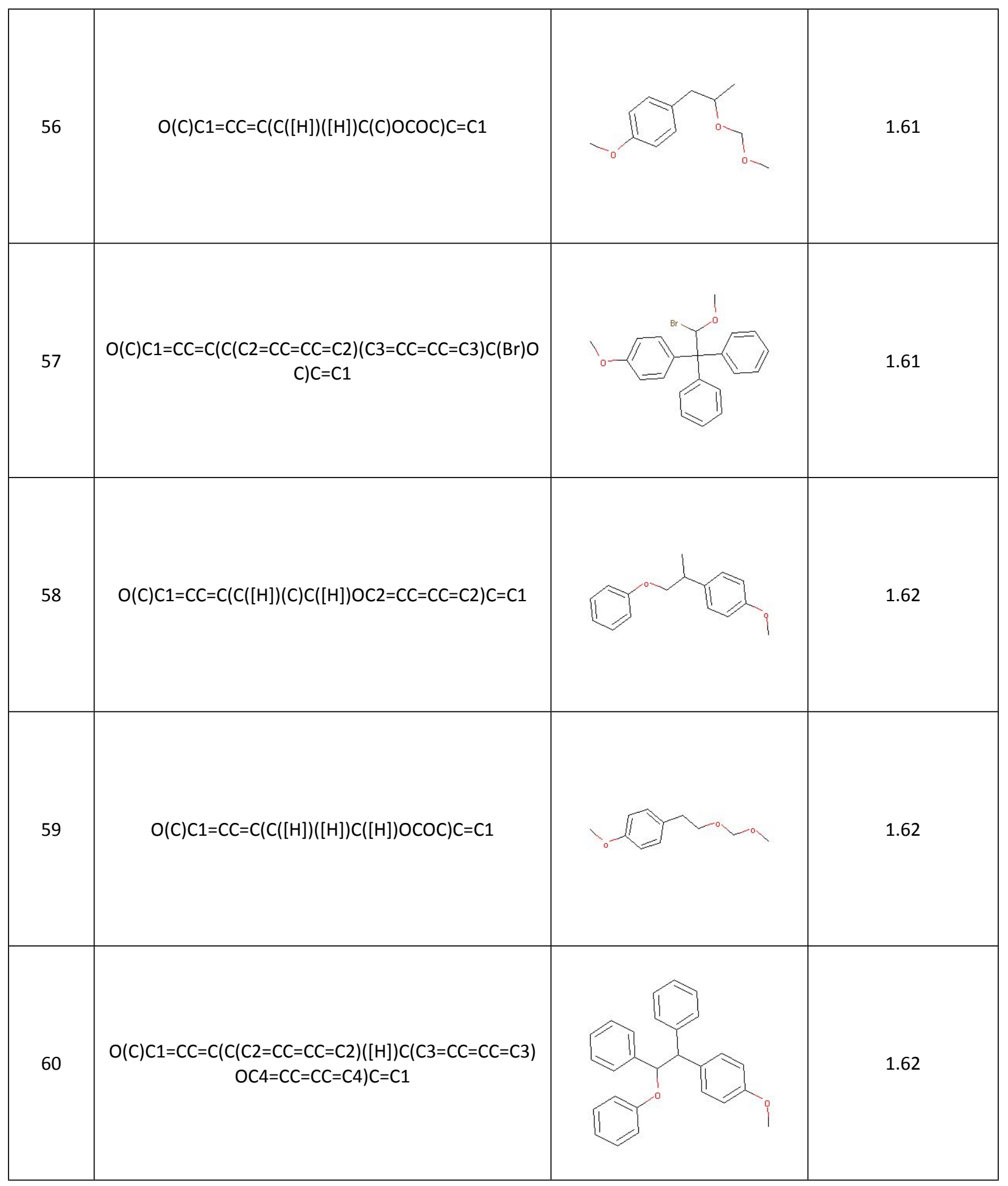

S13 


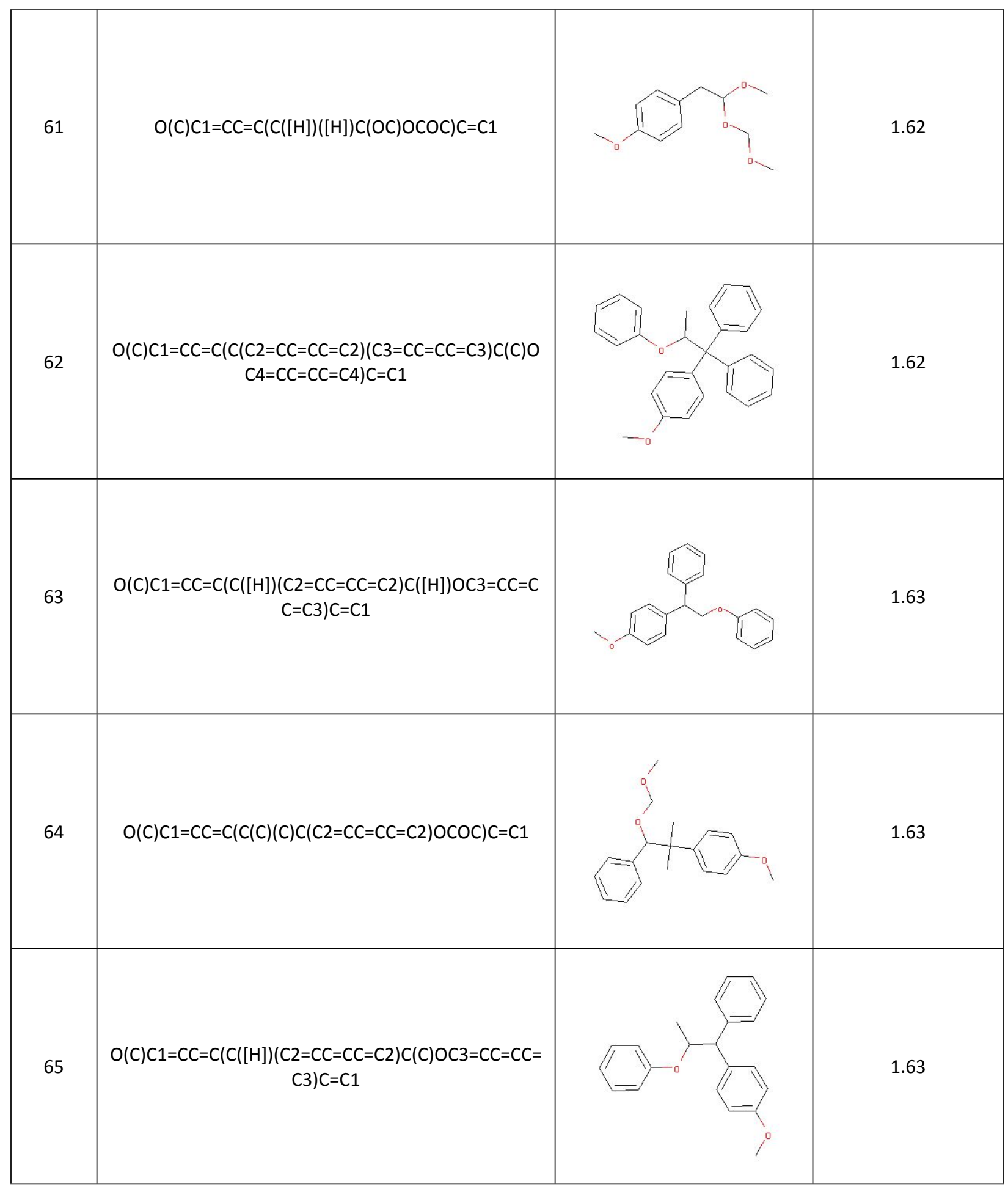




\begin{tabular}{|c|c|c|}
\hline 66 & $\begin{aligned} \mathrm{O}(\mathrm{C}) \mathrm{C} 1=\mathrm{CC}=\mathrm{C}(\mathrm{C}(\mathrm{C} 2=\mathrm{CC}=\mathrm{CC}=\mathrm{C} 2)(\mathrm{C} 3=\mathrm{CC}=\mathrm{CC}=\mathrm{C} 3) \mathrm{C}(\mathrm{Br}) \mathrm{O} \\
\operatorname{COC}) \mathrm{C}=\mathrm{C} 1\end{aligned}$ & 1.63 \\
\hline 67 & $\begin{aligned} \mathrm{O}(\mathrm{C}) \mathrm{C} 1=\mathrm{CC}=\mathrm{C}(\mathrm{C}(\mathrm{C} 2=\mathrm{CC} & =\mathrm{CC}=\mathrm{C} 2)([\mathrm{H}]) \mathrm{C}(\mathrm{OC}) \mathrm{OC} 3=\mathrm{CC}=\mathrm{CC} \\
& =\mathrm{C} 3) \mathrm{C}=\mathrm{C} 1\end{aligned}$ & 1.63 \\
\hline 68 & $\begin{aligned} \mathrm{O}(\mathrm{C}) \mathrm{C} 1=\mathrm{CC}=\mathrm{C}(\mathrm{C}([\mathrm{H}])(\mathrm{C} 2=\mathrm{CC}=\mathrm{CC}=\mathrm{C} 2) \mathrm{C}(\mathrm{Br}) \mathrm{OC} 3=\mathrm{CC}=\mathrm{CC} \\
=\mathrm{C} 3) \mathrm{C}=\mathrm{C} 1\end{aligned}$ & 1.63 \\
\hline 69 & $\mathrm{O}(\mathrm{C}) \mathrm{C} 1=\mathrm{CC}=\mathrm{C}(\mathrm{C}([\mathrm{H}])(\mathrm{C}) \mathrm{C}(\mathrm{Br}) \mathrm{OC}) \mathrm{C}=\mathrm{C} 1$ & 1.64 \\
\hline 70 & $\mathrm{O}(\mathrm{C}) \mathrm{C} 1=\mathrm{CC}=\mathrm{C}(\mathrm{C}(\mathrm{C} 2=\mathrm{CC}=\mathrm{CC}=\mathrm{C} 2)(\mathrm{C}) \mathrm{C}(\mathrm{Br}) \mathrm{OC}) \mathrm{C}=\mathrm{C} 1$ & 1.64 \\
\hline
\end{tabular}




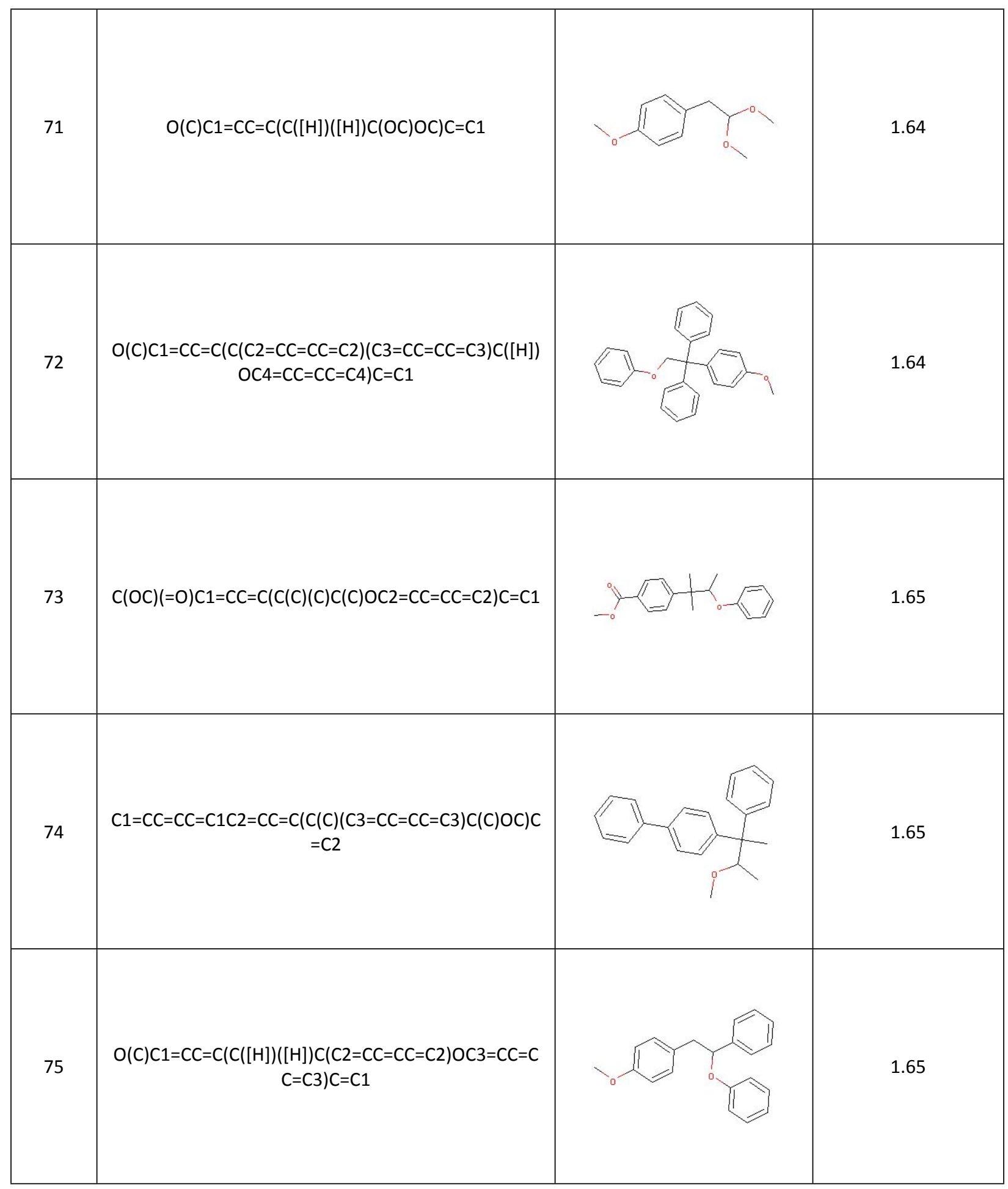




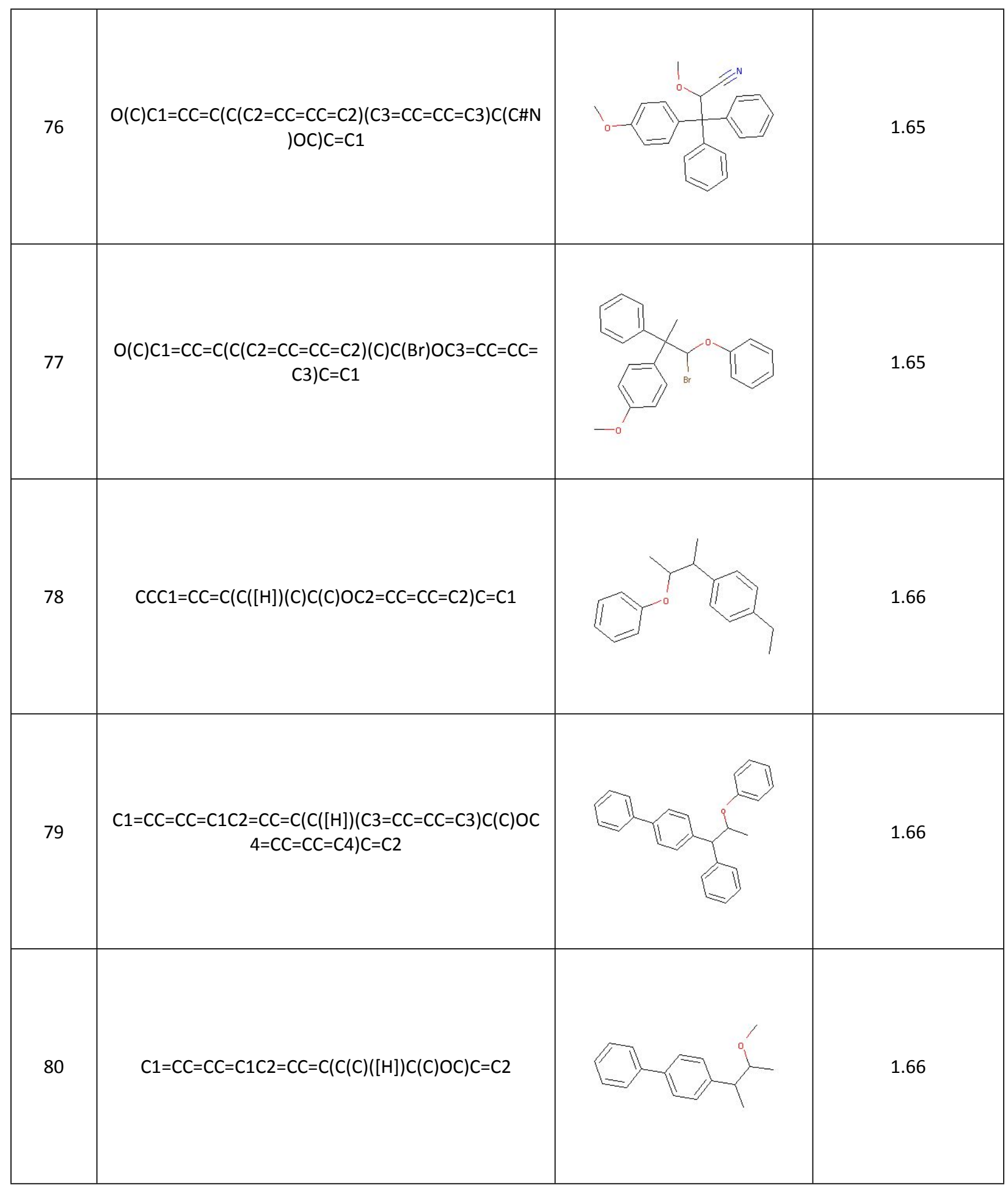




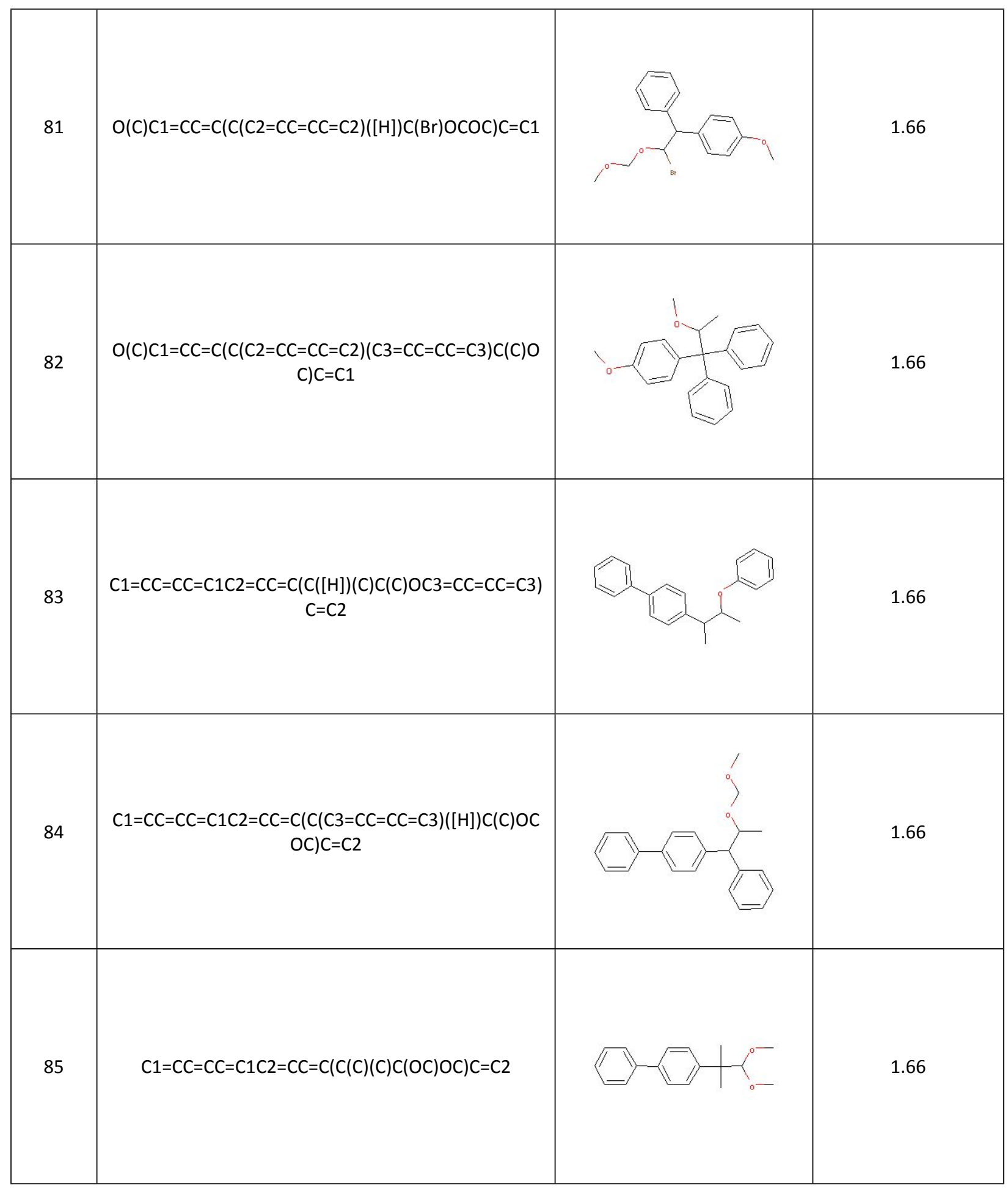




\begin{tabular}{|c|c|c|}
\hline 86 & $\begin{array}{c}\mathrm{C} 1=\mathrm{CC}=\mathrm{CC}=\mathrm{C} 1 \mathrm{C} 2=\mathrm{CC}=\mathrm{C}(\mathrm{C}(\mathrm{C} 3=\mathrm{CC}=\mathrm{CC}=\mathrm{C} 3)(\mathrm{C}) \mathrm{C}(\mathrm{OC}) \mathrm{OC}) \\
\mathrm{C}=\mathrm{C} 2\end{array}$ & 1.67 \\
\hline 87 & $\begin{aligned} \mathrm{C} 1=\mathrm{CC}=\mathrm{CC}=\mathrm{C} 1 \mathrm{C} 2=\mathrm{CC}=\mathrm{C}(\mathrm{C}(\mathrm{C} 3=\mathrm{CC}=\mathrm{CC}=\mathrm{C} 3)(\mathrm{C}) \mathrm{C}(\mathrm{OC}) \mathrm{OC} \\
\mathrm{OC}) \mathrm{C}=\mathrm{C} 2\end{aligned}$ & 1.67 \\
\hline 88 & $\begin{array}{c}\mathrm{O}(\mathrm{C}) \mathrm{C} 1=\mathrm{CC}=\mathrm{C}(\mathrm{C}(\mathrm{C} 2=\mathrm{CC}=\mathrm{CC}=\mathrm{C} 2)(\mathrm{C} 3=\mathrm{CC}=\mathrm{CC}=\mathrm{C} 3) \mathrm{C}(\mathrm{C} 4= \\
\mathrm{CC}=\mathrm{CC}=\mathrm{C} 4) \mathrm{OCOC}) \mathrm{C}=\mathrm{C} 1\end{array}$ & 1.67 \\
\hline 89 & 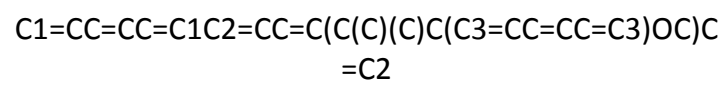 & 1.67 \\
\hline 90 & $\mathrm{O}(\mathrm{C}) \mathrm{C} 1=\mathrm{CC}=\mathrm{C}(\mathrm{C}([\mathrm{H}])([\mathrm{H}]) \mathrm{C}(\mathrm{Br}) \mathrm{OCOC}) \mathrm{C}=\mathrm{C} 1$ & 1.67 \\
\hline
\end{tabular}




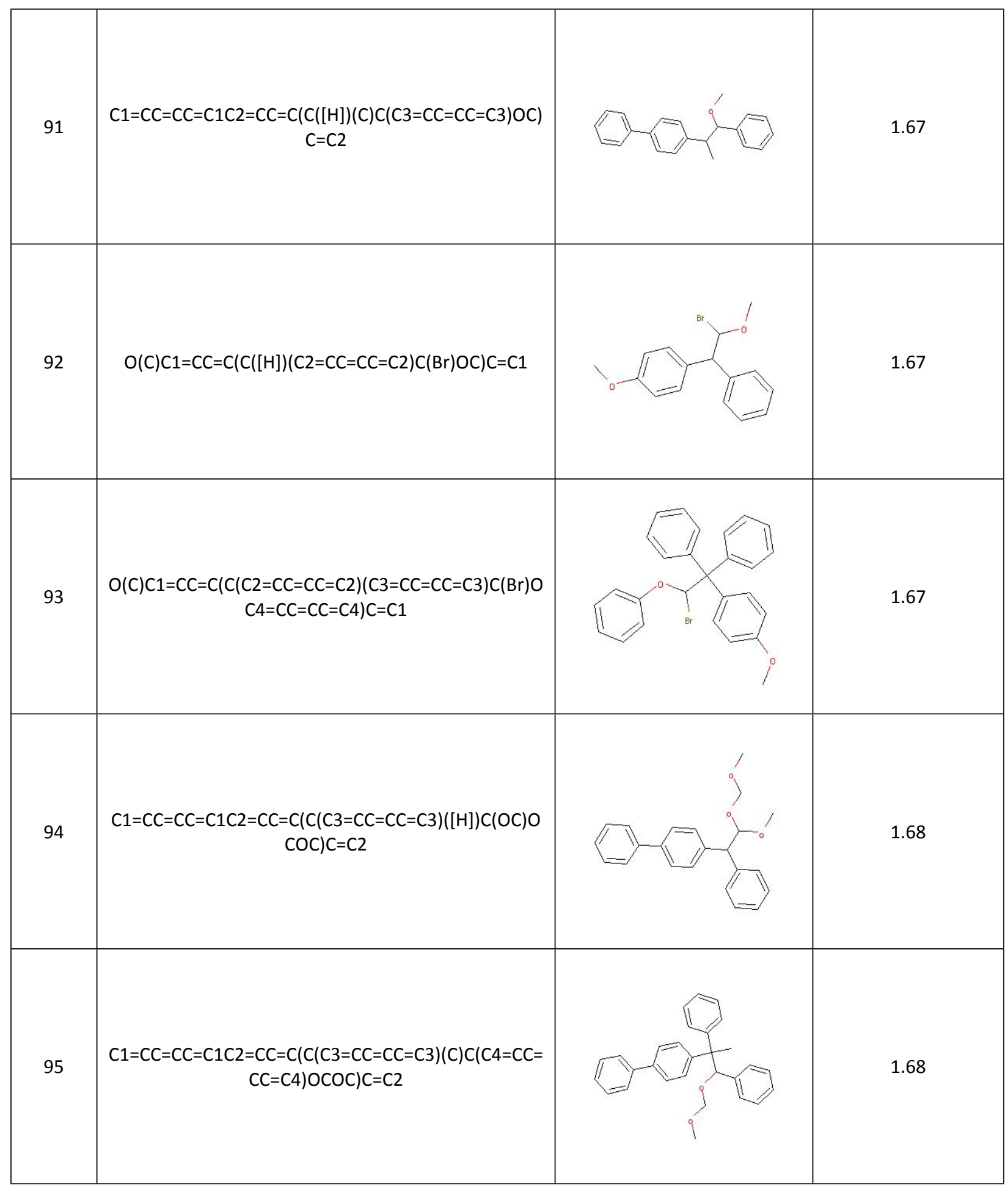




\begin{tabular}{|c|c|c|}
\hline 96 & $\begin{array}{c}\mathrm{O}(\mathrm{C}) \mathrm{C} 1=\mathrm{CC}=\mathrm{C}(\mathrm{C}(\mathrm{C} 2=\mathrm{CC}=\mathrm{CC}=\mathrm{C} 2)(\mathrm{C} 3=\mathrm{CC}=\mathrm{CC}=\mathrm{C} 3) \mathrm{C}(\mathrm{OC}) \\
\mathrm{OC} 4=\mathrm{CC}=\mathrm{CC}=\mathrm{C} 4) \mathrm{C}=\mathrm{C} 1\end{array}$ & 1.68 \\
\hline 97 & $\begin{array}{c}\mathrm{C} 1=\mathrm{CC}=\mathrm{CC}=\mathrm{C} 1 \mathrm{C} 2=\mathrm{CC}=\mathrm{C}(\mathrm{C}(\mathrm{C} 3=\mathrm{CC}=\mathrm{CC}=\mathrm{C} 3)([\mathrm{H}]) \mathrm{C}(\mathrm{OC}) \mathrm{O} \\
\mathrm{C}) \mathrm{C}=\mathrm{C} 2\end{array}$ & 1.68 \\
\hline 98 & $\mathrm{C} 1=\mathrm{CC}=\mathrm{CC}=\mathrm{C} 1 \mathrm{C} 2=\mathrm{CC}=\mathrm{C}(\mathrm{C}([\mathrm{H}])(\mathrm{C}) \mathrm{C}(\mathrm{C}) \mathrm{OCOC}) \mathrm{C}=\mathrm{C} 2$ & 1.68 \\
\hline 99 & $\mathrm{O}(\mathrm{C}) \mathrm{C} 1=\mathrm{CC}=\mathrm{C}(\mathrm{C}([\mathrm{H}])([\mathrm{H}]) \mathrm{C}([\mathrm{H}]) \mathrm{OC} 2=\mathrm{CC}=\mathrm{CC}=\mathrm{C} 2) \mathrm{C}=\mathrm{C} 1$ & 1.68 \\
\hline 100 & $\begin{array}{c}\mathrm{C} 1=\mathrm{CC}=\mathrm{CC}=\mathrm{C} 1 \mathrm{C} 2=\mathrm{CC}=\mathrm{C}(\mathrm{C}(\mathrm{C})(\mathrm{C}) \mathrm{C}(\mathrm{C} 3=\mathrm{CC}=\mathrm{CC}=\mathrm{C} 3) \mathrm{OCO} \\
\mathrm{C}) \mathrm{C}=\mathrm{C} 2\end{array}$ & 1.68 \\
\hline
\end{tabular}




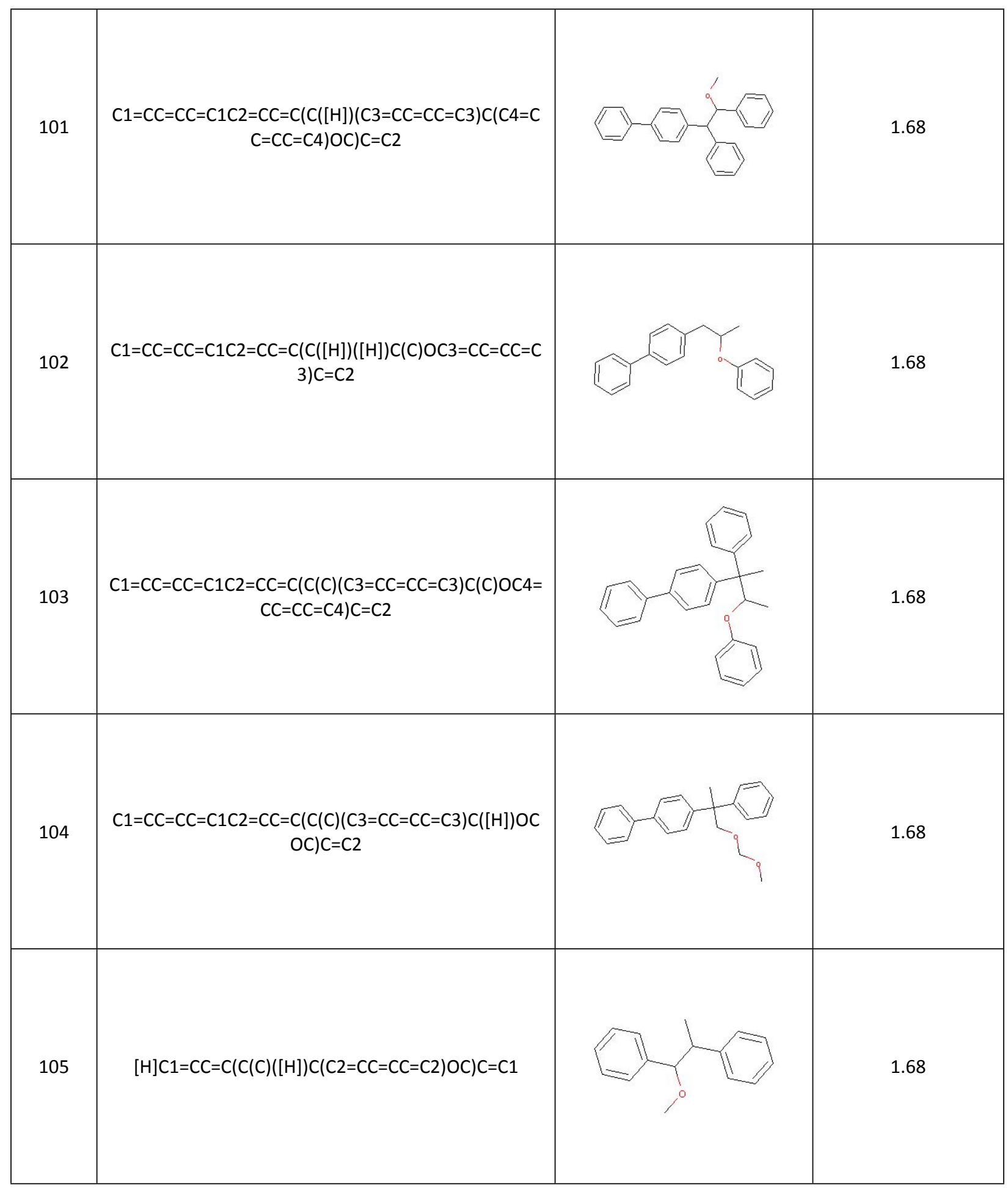




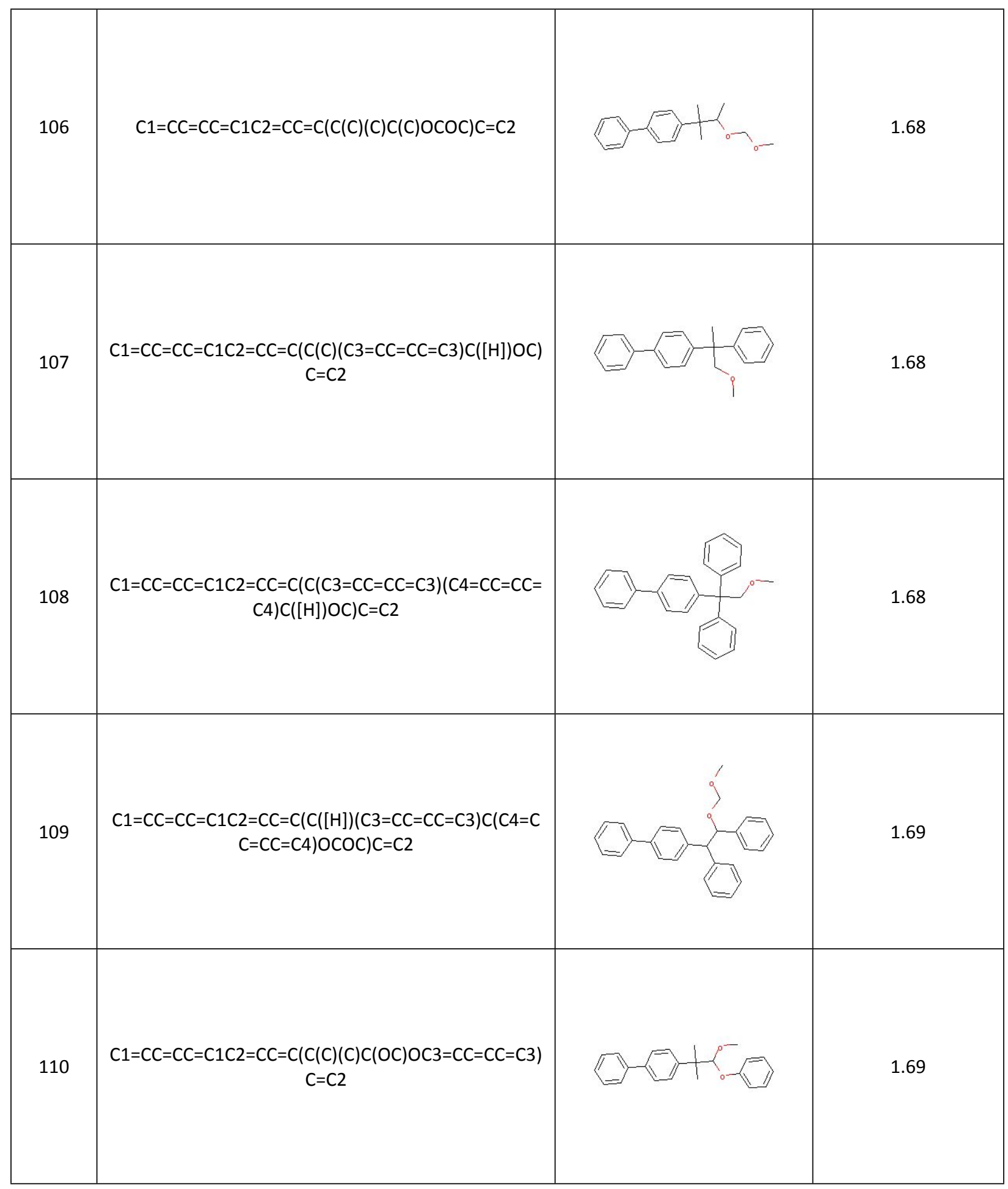




\begin{tabular}{|c|c|c|}
\hline 111 & $\begin{array}{c}\mathrm{C} 1=\mathrm{CC}=\mathrm{CC}=\mathrm{C} 1 \mathrm{C} 2=\mathrm{CC}=\mathrm{C}(\mathrm{C}(\mathrm{C} 3=\mathrm{CC}=\mathrm{CC}=\mathrm{C} 3)(\mathrm{C} 4=\mathrm{CC}=\mathrm{CC}= \\
\mathrm{C} 4) \mathrm{C}(\mathrm{C}=\mathrm{C}=\mathrm{CC}=\mathrm{CC}=\mathrm{C} 5) \mathrm{OC}) \mathrm{C}=\mathrm{C} 2\end{array}$ & 1.69 \\
\hline 112 & $\mathrm{O}(\mathrm{C}) \mathrm{C} 1=\mathrm{CC}=\mathrm{C}(\mathrm{C}([\mathrm{H}])(\mathrm{C}) \mathrm{C}(\mathrm{Br}) \mathrm{OCOC}) \mathrm{C}=\mathrm{C} 1$ & 1.69 \\
\hline 113 & $\mathrm{C} 1=\mathrm{CC}=\mathrm{CC}=\mathrm{C} 1 \mathrm{C} 2=\mathrm{CC}=\mathrm{C}(\mathrm{C}(\mathrm{C})(\mathrm{C}) \mathrm{C}([\mathrm{H}]) \mathrm{OC}) \mathrm{C}=\mathrm{C} 2$ & 1.69 \\
\hline 114 & $\begin{aligned} \mathrm{C} 1=\mathrm{CC}=\mathrm{CC}=\mathrm{C} 1 \mathrm{C} 2=\mathrm{CC}=\mathrm{C}(\mathrm{C}(\mathrm{C} 3=\mathrm{CC}=\mathrm{CC}=\mathrm{C} 3)(\mathrm{C}) \mathrm{C}(\mathrm{OC}) \mathrm{OC} \\
4=\mathrm{CC}=\mathrm{CC}=\mathrm{C4}) \mathrm{C}=\mathrm{C} 2\end{aligned}$ & 1.69 \\
\hline 115 & $\begin{array}{c}\mathrm{C}(\# \mathrm{~N}) \mathrm{C} 1=\mathrm{CC}=\mathrm{C}(\mathrm{C}(\mathrm{C})(\mathrm{C}) \mathrm{C}(\mathrm{C} 2=\mathrm{CC}=\mathrm{CC}=\mathrm{C} 2) \mathrm{OC}=\mathrm{CC}=\mathrm{CC}= \\
\mathrm{C} 3) \mathrm{C}=\mathrm{C1}\end{array}$ & 1.69 \\
\hline
\end{tabular}




\begin{tabular}{|c|c|c|}
\hline 116 & $\begin{array}{c}\mathrm{C} 1=\mathrm{CC}=\mathrm{CC}=\mathrm{C} 1 \mathrm{C} 2=\mathrm{CC}=\mathrm{C}(\mathrm{C}(\mathrm{C} 3=\mathrm{CC}=\mathrm{CC}=\mathrm{C} 3)(\mathrm{C} 4=\mathrm{CC}=\mathrm{CC}= \\
\mathrm{C} 4) \mathrm{C}(\mathrm{C} 5=\mathrm{CC}=\mathrm{CC}=\mathrm{C} 5) \mathrm{OCOC}) \mathrm{C}=\mathrm{C} 2\end{array}$ & 1.69 \\
\hline 117 & $\begin{aligned} \mathrm{C} 1=\mathrm{CC}=\mathrm{CC}=\mathrm{C} 1 \mathrm{C} 2=\mathrm{CC}= & \mathrm{C}(\mathrm{C}([\mathrm{H}])([\mathrm{H}]) \mathrm{C}([\mathrm{H}]) \mathrm{OC} 3=\mathrm{CC}=\mathrm{CC}= \\
& \mathrm{C} 3) \mathrm{C}=\mathrm{C} 2\end{aligned}$ & 1.69 \\
\hline 118 & $\mathrm{C} 1=\mathrm{CC}=\mathrm{CC}=\mathrm{C} 1 \mathrm{C} 2=\mathrm{CC}=\mathrm{C}(\mathrm{C}([\mathrm{H}])(\mathrm{C}) \mathrm{C}(\mathrm{OC}) \mathrm{OC}) \mathrm{C}=\mathrm{C} 2$ & 1.69 \\
\hline 119 & $\begin{aligned} & \mathrm{C} 1=\mathrm{CC}=\mathrm{CC}=\mathrm{C} 1 \mathrm{C} 2=\mathrm{CC}==\mathrm{C}(\mathrm{C}(\mathrm{C} 3=\mathrm{CC}=\mathrm{CC}=\mathrm{C} 3)(\mathrm{C}) \mathrm{C}(\mathrm{C} 4=\mathrm{CC}= \\
&\mathrm{CC}=\mathrm{C} 4) \mathrm{OC}) \mathrm{C}=\mathrm{C} 2\end{aligned}$ & 1.69 \\
\hline 120 & $\begin{aligned} \mathrm{C} 1=\mathrm{CC}=\mathrm{CC}=\mathrm{C} 1 \mathrm{C} 2=\mathrm{CC}=\mathrm{C}(\mathrm{C}(\mathrm{C} 3=\mathrm{CC}=\mathrm{CC}=\mathrm{C} 3)(\mathrm{C} 4=\mathrm{CC}=\mathrm{CC}= \\
\mathrm{C} 4) \mathrm{C}(\mathrm{OC}) \mathrm{OC}) \mathrm{C}=\mathrm{C} 2\end{aligned}$ & 1.69 \\
\hline
\end{tabular}




\begin{tabular}{|c|c|c|}
\hline 121 & $\begin{array}{c}\mathrm{O}(\mathrm{C}) \mathrm{C} 1=\mathrm{CC}=\mathrm{C}(\mathrm{C}(\mathrm{C} 2=\mathrm{CC}=\mathrm{CC}=\mathrm{C} 2)(\mathrm{C} 3=\mathrm{CC}=\mathrm{CC}=\mathrm{C} 3) \mathrm{C}(\mathrm{CHN} \\
) \mathrm{OC} 4=\mathrm{CC}=\mathrm{CC}=\mathrm{C} 4) \mathrm{C}=\mathrm{C} 1\end{array}$ & 1.69 \\
\hline 122 & {$[\mathrm{H}] \mathrm{C} 1=\mathrm{CC}=\mathrm{C}(\mathrm{C}(\mathrm{C})(\mathrm{C}) \mathrm{C}(\mathrm{C}) \mathrm{OC} 2=\mathrm{CC}=\mathrm{CC}=\mathrm{C} 2) \mathrm{C}=\mathrm{C} 1$} & 1.69 \\
\hline 123 & $\mathrm{C} 1=\mathrm{CC}=\mathrm{CC}=\mathrm{C} 1 \mathrm{C} 2=\mathrm{CC}=\mathrm{C}(\mathrm{C}([\mathrm{H}])([\mathrm{H}]) \mathrm{C}(\mathrm{C}) \mathrm{OC}) \mathrm{C}=\mathrm{C} 2$ & 1.69 \\
\hline 124 & $\begin{array}{c}\mathrm{C} 1=\mathrm{CC}=\mathrm{CC}=\mathrm{C} 1 \mathrm{C} 2=\mathrm{CC}=\mathrm{C}(\mathrm{C}(\mathrm{C})(\mathrm{C} 3=\mathrm{CC}=\mathrm{CC}=\mathrm{C} 3) \mathrm{C}(\mathrm{C}) \mathrm{OCO} \\
\mathrm{C}) \mathrm{C}=\mathrm{C} 2\end{array}$ & 1.69 \\
\hline 125 & $\mathrm{C} 1=\mathrm{CC}=\mathrm{CC}=\mathrm{C} 1 \mathrm{C} 2=\mathrm{CC}=\mathrm{C}(\mathrm{C}(\mathrm{C})(\mathrm{C}) \mathrm{C}(\mathrm{C}) \mathrm{OC}) \mathrm{C}=\mathrm{C} 2$ & 1.69 \\
\hline
\end{tabular}




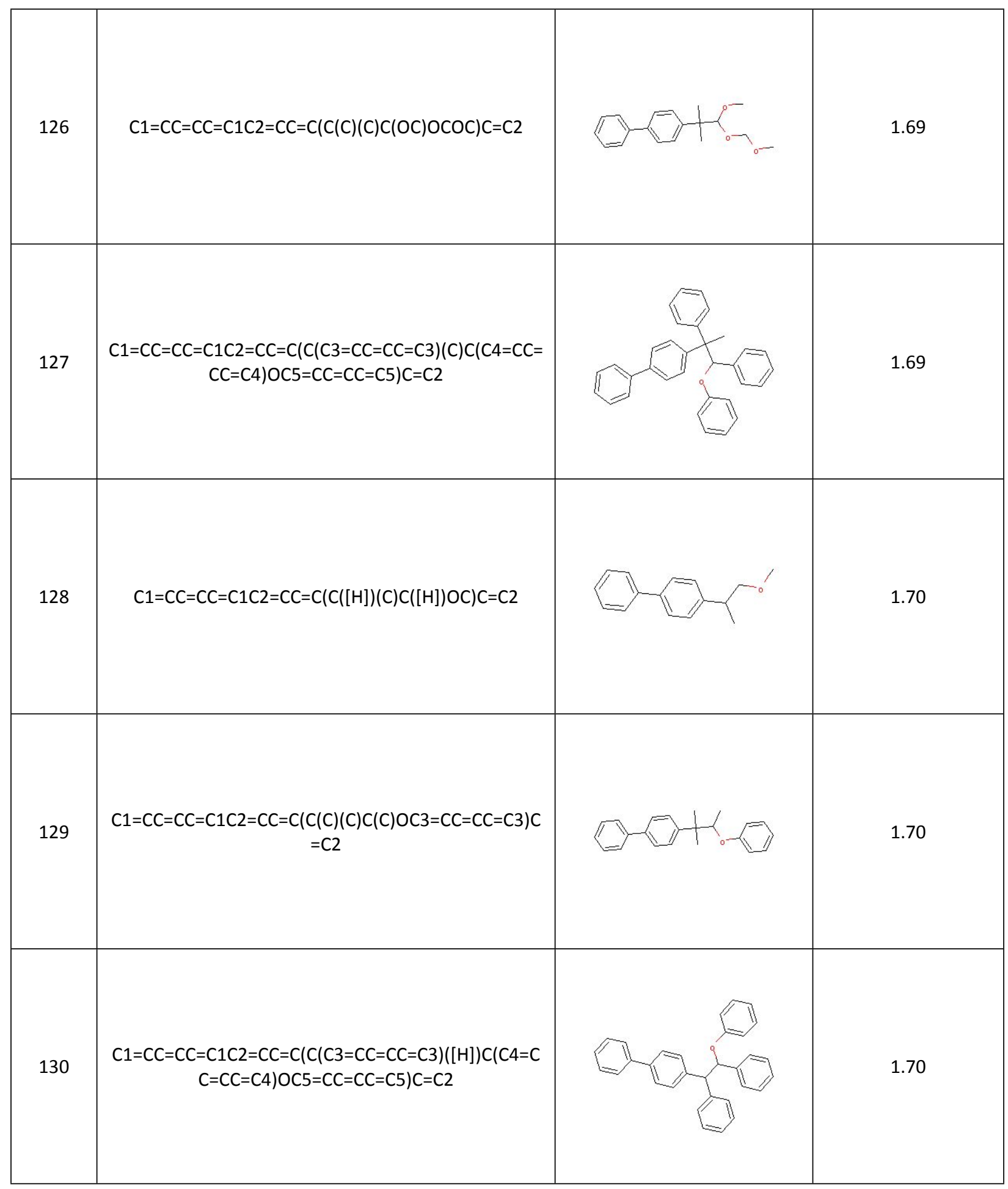




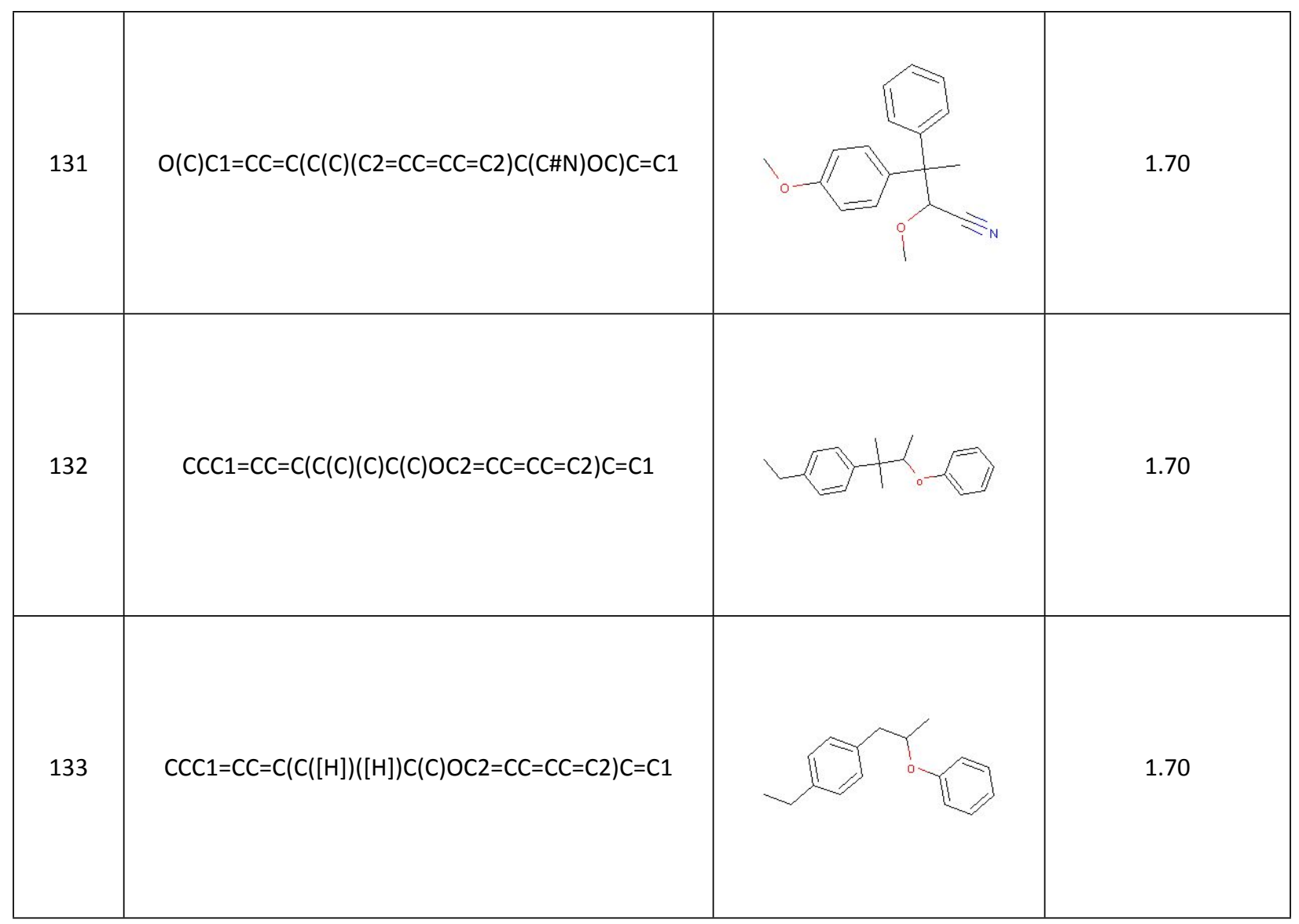


Table S2 Most favorable mesolytic cleavage pathways (red bonds) and their corresponding fragmentation free energy ( $\Delta G_{\mathrm{min}}^{\mathrm{meso}}$ ) for 10 randomly selected molecules from the 133 identified HBEs in Table S1.

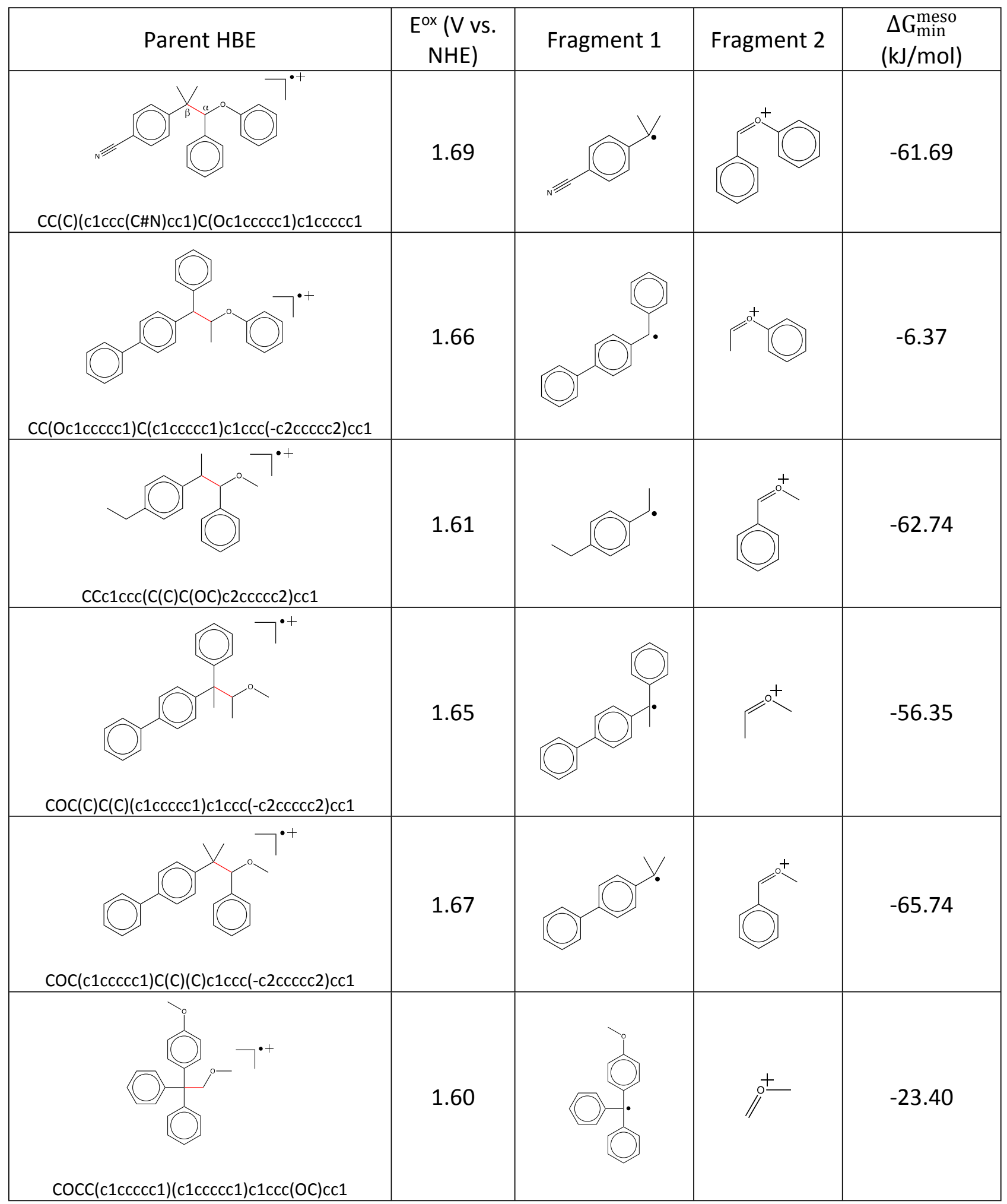




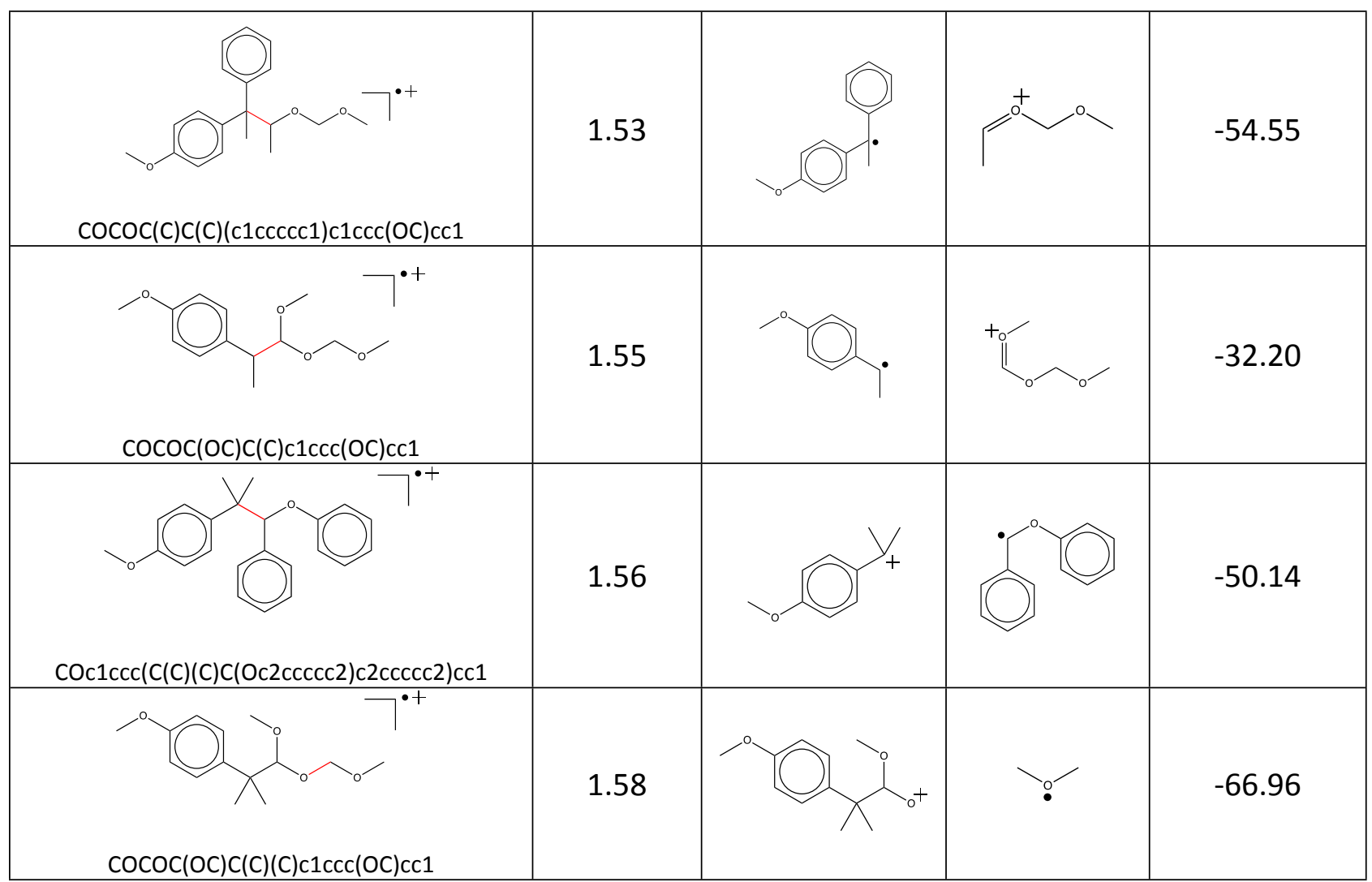


Table S3 List of 49 tabulated features and their value ranges. The Pearson correlation coefficients were derived from DFT-computed oxidation potentials.

\begin{tabular}{|c|c|c|c|c|c|}
\hline Feature & Comment & Unit & Min & Max & $\begin{array}{l}\text { Pearson } \\
\text { correlation } \\
\text { coefficient }\end{array}$ \\
\hline sssr & Smallest set of smallest rings & & 1.0 & 6.0 & -0.30 \\
\hline clogp ${ }^{1}$ & $\begin{array}{l}\text { Crippen's Partition Coefficient } \\
\text { (measure of lipophilicity) }\end{array}$ & & 1.27 & 9.51 & -0.36 \\
\hline Molar refractivity $(\mathrm{mr})^{1}$ & & $\mathrm{~m}^{3} / \mathrm{mol}$ & 42.02 & 160.9 & -0.36 \\
\hline Molecular weight $(\mathrm{mw})$ & & $\mathrm{g} / \mathrm{mol}$ & 136.09 & 504.11 & -0.20 \\
\hline tpsa $^{2}$ & Topological polar surface area & $\AA^{2}$ & 9.23 & 132.97 & 0.57 \\
\hline chiOn $^{3}$ & $\begin{array}{l}\text { Simple molecular connectivity } \\
\text { index (order 0) }\end{array}$ & & 6.21 & 21.73 & -0.36 \\
\hline chi1n ${ }^{3}$ & $\begin{array}{l}\text { Simple molecular connectivity } \\
\text { index (order 1) }\end{array}$ & & 3.46 & 13.39 & -0.34 \\
\hline $\operatorname{chi} 2 \mathrm{n}^{3}$ & $\begin{array}{l}\text { Simple molecular connectivity } \\
\text { index (order } 2 \text { ) }\end{array}$ & & 2.23 & 9.71 & -0.41 \\
\hline chi3n ${ }^{3}$ & $\begin{array}{l}\text { Simple molecular connectivity } \\
\text { index (order } 3 \text { ) }\end{array}$ & & 1.44 & 7.4 & -0.33 \\
\hline chi4n ${ }^{3}$ & $\begin{array}{l}\text { Simple molecular connectivity } \\
\text { index (order 4) }\end{array}$ & & 0.86 & 5.58 & -0.30 \\
\hline chiOv $^{3}$ & $\begin{array}{l}\text { Valence molecular connectivity } \\
\text { index (order } 0 \text { ) }\end{array}$ & & 6.21 & 21.73 & -0.34 \\
\hline $\operatorname{chi1v}^{3}$ & $\begin{array}{l}\text { Valence molecular connectivity } \\
\text { index (order 1) }\end{array}$ & & 3.46 & 13.39 & -0.33 \\
\hline chi2 $2 v^{3}$ & $\begin{array}{l}\text { Valence molecular connectivity } \\
\text { index (order 2) }\end{array}$ & & 2.23 & 9.71 & -0.39 \\
\hline chi3v $v^{3}$ & $\begin{array}{l}\text { Valence molecular connectivity } \\
\text { index (order 3) }\end{array}$ & & 1.44 & 7.4 & -0.30 \\
\hline chi4v $v^{3}$ & $\begin{array}{l}\text { Valence molecular connectivity } \\
\text { index (order 4) }\end{array}$ & & 0.86 & 5.58 & -0.28 \\
\hline fracsp3 & Fraction of $\mathrm{sp3} \mathrm{C}$ & & 0.05 & 0.6 & 0.03 \\
\hline hall_kier_alpha ${ }^{3}$ & $\begin{array}{l}\text { Ratio of covalent radii of atom } \\
(\mathrm{x}) \text { and } \mathrm{sp} 3 \mathrm{C} \text { atom }\left(\alpha_{x}=\right. \\
\left.\left(r_{x} / r_{C_{s p 3}}\right)-1\right)\end{array}$ & & -4.88 & -0.34 & 0.05 \\
\hline kappa1 ${ }^{3}$ & Molecular shape index (order 1) & & 7.29 & 24.46 & -0.22 \\
\hline kappa $2^{3}$ & Molecular shape index (order 2) & & 3.72 & 11.25 & -0.21 \\
\hline kappa3 $^{3}$ & Molecular shape index (order 3) & & 1.73 & 4.88 & -0.32 \\
\hline $\begin{array}{l}\text { Labute's approximate surface area } \\
\text { (labuteasa) }\end{array}$ & & $\AA^{2}$ & 61.64 & 230.56 & -0.26 \\
\hline number_aromatic_rings & & & 1.0 & 6.0 & -0.30 \\
\hline number_atom_stereocenters & & & 0.0 & 2.0 & -0.02 \\
\hline number_HBA & & & 1.0 & 7.0 & 0.36 \\
\hline number_hetero_atoms & & & 1.0 & 8.0 & 0.41 \\
\hline number_rings & & & 1.0 & 6.0 & -0.30 \\
\hline number_rotatable_bonds & & & 3.0 & 9.0 & -0.23 \\
\hline number_heavy_atoms & & & 10.0 & 39.0 & -0.23 \\
\hline number_n_o & & & 1.0 & 8.0 & 0.39 \\
\hline number_valence_electrons & & & 54.0 & 188.0 & -0.27 \\
\hline max_partial_charge & & & 0.05 & 0.34 & 0.40 \\
\hline
\end{tabular}




\begin{tabular}{|l|l|l|l|l|l|}
\hline min_partial_charge & & -0.5 & -0.35 & 0.22 \\
\hline fr_C_O & Number of -CO & & 0.0 & 1.0 & 0.24 \\
\hline fr_C_O_noCOO & & & 0.0 & 1.0 & 0.24 \\
\hline fr_methoxy & Number of -OCH3 & & 0.0 & 3.0 & 0.17 \\
\hline fr_ester & & & 0.0 & 1.0 & 0.24 \\
\hline fr_ether & & & 1.0 & 4.0 & 0.10 \\
\hline fr_NHO & & 0.0 & 4.0 & 0.21 \\
\hline fr_aniline & & 0.0 & 1.0 & -0.76 \\
\hline fr_nitrile & & & 0.0 & 4.0 & 0.41 \\
\hline fr_nitro & & & 0.0 & 1.0 & 0.30 \\
\hline fr_halogen & & & 0.0 & 1.0 & 0.06 \\
\hline fr_alkyl_halide & & 0.0 & 1.0 & 0.06 \\
\hline fr_unbrch_alkane & Number of unbranched alkane & & 0.0 & 2.0 & 0.04 \\
\hline fr_benzene & & & 1.0 & 6.0 & -0.30 \\
\hline fr_nitro_arom & & 0.0 & 1.0 & 0.30 \\
\hline fr_nitro_arom_nonortho & & & 0.0 & 1.0 & 0.30 \\
\hline fr_para_hydroxylation & & & 0.0 & 1.0 & -0.24 \\
\hline fr_aryl_methyl & & & 0.0 & 1.0 & 0.06 \\
\hline
\end{tabular}

Table S4 List of functional groups and their corresponding scaffold sites used for populating HBE database of 112,000 entries

\begin{tabular}{|l|l|}
\hline Scaffold Site & Functional group \\
\hline R1 & $-\mathrm{Me},-\mathrm{Et},-\mathrm{Pr},-\mathrm{Ph},-\mathrm{COMe},-\mathrm{C}(\mathrm{Me}) \mathrm{Me},-\mathrm{EtOMe}$ \\
\hline R2 & $\begin{array}{l}-\mathrm{N}(\mathrm{Me})_{3}{ }^{+},-\mathrm{COMe},-\mathrm{Et},-\mathrm{OCMe}, \mathrm{N}(\mathrm{Me})_{2},-\mathrm{NO}_{2},-\mathrm{C}(=\mathrm{O}),-\mathrm{Pr},-\mathrm{PPr},- \\
\mathrm{EtOMe},-\mathrm{C}(\mathrm{Me}) \mathrm{OMe}\end{array}$ \\
\hline R3 & $\begin{array}{l}-\mathrm{N}(\mathrm{Me})_{3}{ }^{+},-\mathrm{Me},-\mathrm{COMe},-\mathrm{Br},-\mathrm{C}(=\mathrm{O}),-\mathrm{OEth},-\mathrm{Pr},-\mathrm{PPr},-\mathrm{C}(\mathrm{COMe}),- \\
\mathrm{C}(\mathrm{Me})(\mathrm{OMe}),-\mathrm{OPr},-\mathrm{O} P \mathrm{Pr}\end{array}$ \\
\hline R4 & $\begin{array}{l}-\mathrm{N}(\mathrm{Me})_{3}{ }^{+},-\mathrm{OMe},-\mathrm{COMe},-\mathrm{Br},-\mathrm{N}(\mathrm{Me}) \mathrm{Me},-\mathrm{Et},-\mathrm{OEt},-\mathrm{NO}_{2},-\mathrm{C}(=\mathrm{O}),-\mathrm{Pr}, \\
-\mathrm{C}(\mathrm{Me}) \mathrm{Me},-\mathrm{C}(\mathrm{COMe}),-\mathrm{C}(\mathrm{Me})(\mathrm{OMe}),-\mathrm{OPr},-\mathrm{OiPr})\end{array}$ \\
\hline R5 & $\begin{array}{l}-\mathrm{N}(\mathrm{Me})_{3}{ }^{+},-\mathrm{OMe},-\mathrm{COMe},-\mathrm{Br},-\mathrm{N}(\mathrm{Me}) \mathrm{Me},-\mathrm{Et},-\mathrm{OEt},-\mathrm{NO}_{2},-\mathrm{C}(=\mathrm{O}),-\mathrm{Pr}, \\
-\mathrm{C}(\mathrm{Me}) \mathrm{Me},-\mathrm{C}(\mathrm{COMe}),-\mathrm{C}(\mathrm{Me})(\mathrm{OMe}),-\mathrm{OPr},-\mathrm{OiPr})\end{array}$ \\
\hline
\end{tabular}

Substituent groups are, Ph: phenyl, Et :ethyl, OMe: methoxy, iPr: isopropyl, OPr: propoxy, EtOMe: methoxy ethyl, Pr: propyl 
Table S5 List of HBEs evaluated during BO run \#1 (BO1) and their corresponding Ex. The initial 10 were randomly selected.

\begin{tabular}{|c|c|c|c|c|}
\hline HBE \# & SMILES & 2-D Structure & $\begin{array}{l}\text { DFT-computed } \\
\text { Eox (V vs. NHE) }\end{array}$ & $\begin{array}{c}\text { BO-predicted } \\
\text { Ex (V vs. NHE) }\end{array}$ \\
\hline $1^{\prime}$ & $\begin{array}{l}\mathrm{CCCOC}(\mathrm{OCOC}) \mathrm{C}(\mathrm{CCC})(\mathrm{O} \\
\mathrm{C}) \mathrm{c} 1 \mathrm{ccc}([\mathrm{N}+](\mathrm{C})(\mathrm{C}) \mathrm{C}) \mathrm{cc} 1\end{array}$ & & 1.14 & \\
\hline $2^{\prime}$ & $\begin{array}{c}\mathrm{CCC}(\mathrm{OC})(\mathrm{c} 1 \mathrm{ccc}(\mathrm{C}(\mathrm{C}) \mathrm{C}) \mathrm{cc} \\
1) \mathrm{C}(\mathrm{COC}) \mathrm{OC}(\mathrm{C}) \mathrm{C}\end{array}$ & & 1.87 & \\
\hline $3^{\prime}$ & $\begin{array}{l}\mathrm{COCCc} 1 \mathrm{ccc}(\mathrm{C}(\mathrm{COC})(\mathrm{CO} \\
\mathrm{C}) \mathrm{C}(\mathrm{OC}(\mathrm{C}) \mathrm{C}) \mathrm{OC}(\mathrm{C}) \mathrm{C}) \mathrm{cc} 1\end{array}$ & & 1.87 & \\
\hline $4^{\prime}$ & $\begin{array}{c}\mathrm{CCOC}(\mathrm{OCC})(\mathrm{c} 1 \mathrm{ccc}(\mathrm{CCO} \\
\mathrm{C}) \mathrm{cc} 1) \mathrm{C}(\mathrm{OCCOC})[\mathrm{N}+](= \\
\text { O)[O-] }\end{array}$ & & 2.23 & \\
\hline $5^{\prime}$ & $\begin{array}{c}\mathrm{CCCC}(\mathrm{OC}(\mathrm{C}) \mathrm{C})(\mathrm{c} 1 \mathrm{ccc}(\mathrm{CC} \\
\mathrm{COCC}) \mathrm{cc} 1) \mathrm{C}(\mathrm{COC}) \mathrm{Oc} 1 \mathrm{cc} \\
\mathrm{ccc} 1\end{array}$ & & 1.63 & \\
\hline
\end{tabular}




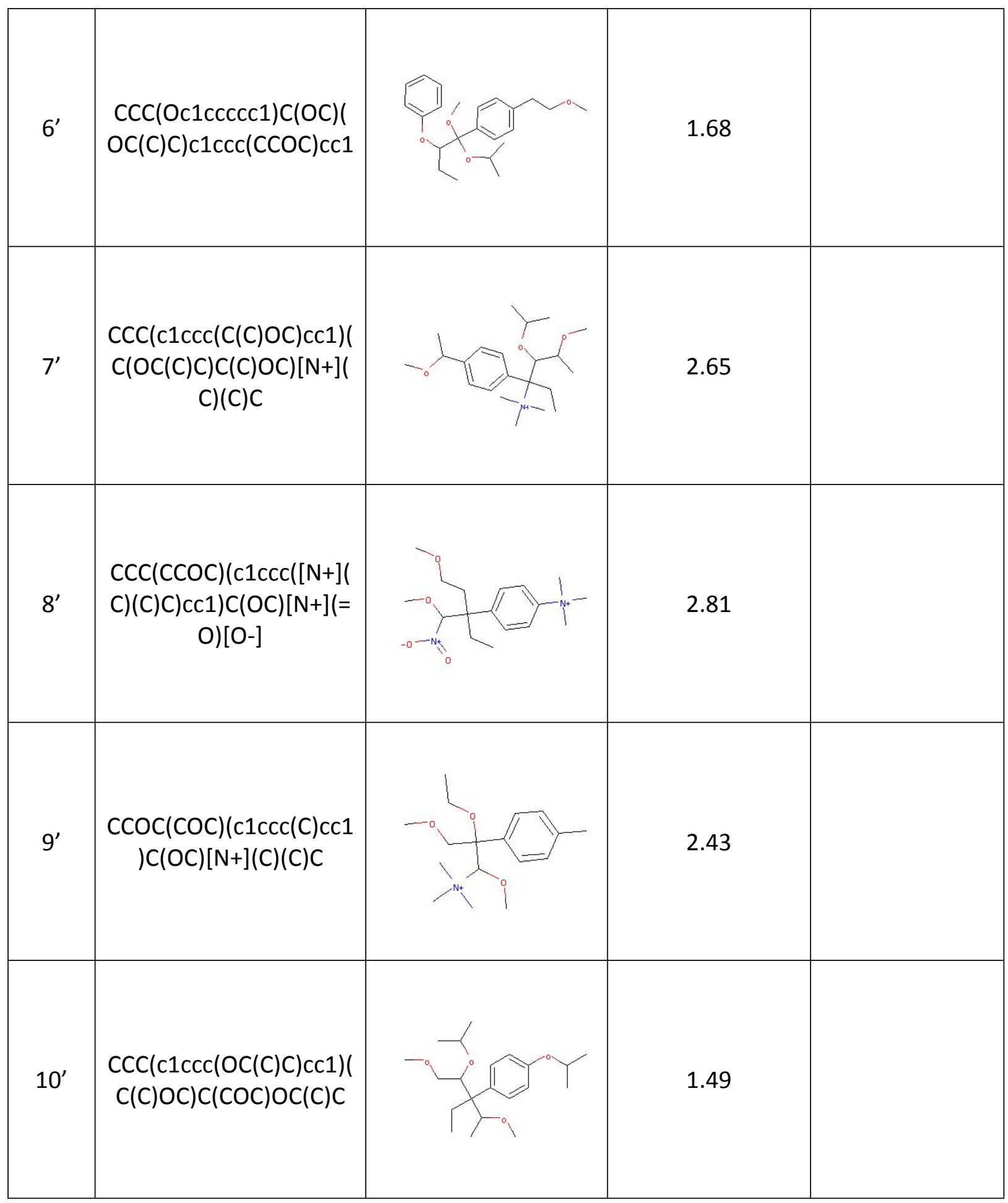




\begin{tabular}{|c|c|c|c|c|}
\hline 1 & $\begin{array}{c}\operatorname{cOC}(\mathrm{C}) \mathrm{c} 1 \mathrm{ccc}(\mathrm{C}(\mathrm{OC}(\mathrm{C}) \mathrm{C}) \\
(\mathrm{C}(\mathrm{C}) \mathrm{OC}) \mathrm{C}(\mathrm{Oc} 2 \mathrm{ccccc} 2) \mathrm{C} \\
\text { (C)OC)cc1 }\end{array}$ & & 1.62 & 1.62 \\
\hline 2 & $\begin{array}{c}\operatorname{cccOc} 1 \operatorname{ccc}(C(C=0)(C=0 \\
) C(C=0) O C(O C) c c 1\end{array}$ & & 1.92 & 1.82 \\
\hline 3 & $\begin{array}{l}C C C O c 1 \operatorname{ccc}(C(C(O C C) N( \\
C) C)(N(C) C) N(C) C) c c 1\end{array}$ & & 0.5 & 1.25 \\
\hline 4 & $\begin{array}{c}\mathrm{CCOC}(\mathrm{OCC}) \mathrm{C}(\mathrm{Br})(\mathrm{OCC}) \mathrm{c} \\
1 \mathrm{ccc}(\mathrm{C}=\mathrm{O}) \mathrm{cc} 1\end{array}$ & & 2.74 & 1.72 \\
\hline 5 & $\begin{array}{c}C C O C C C c 1 \operatorname{ccc}(C(O C C)(C \\
(C) O C) C(O C C O C) C(C) O C \\
) \operatorname{cc} 1\end{array}$ & & 1.78 & 1.76 \\
\hline
\end{tabular}




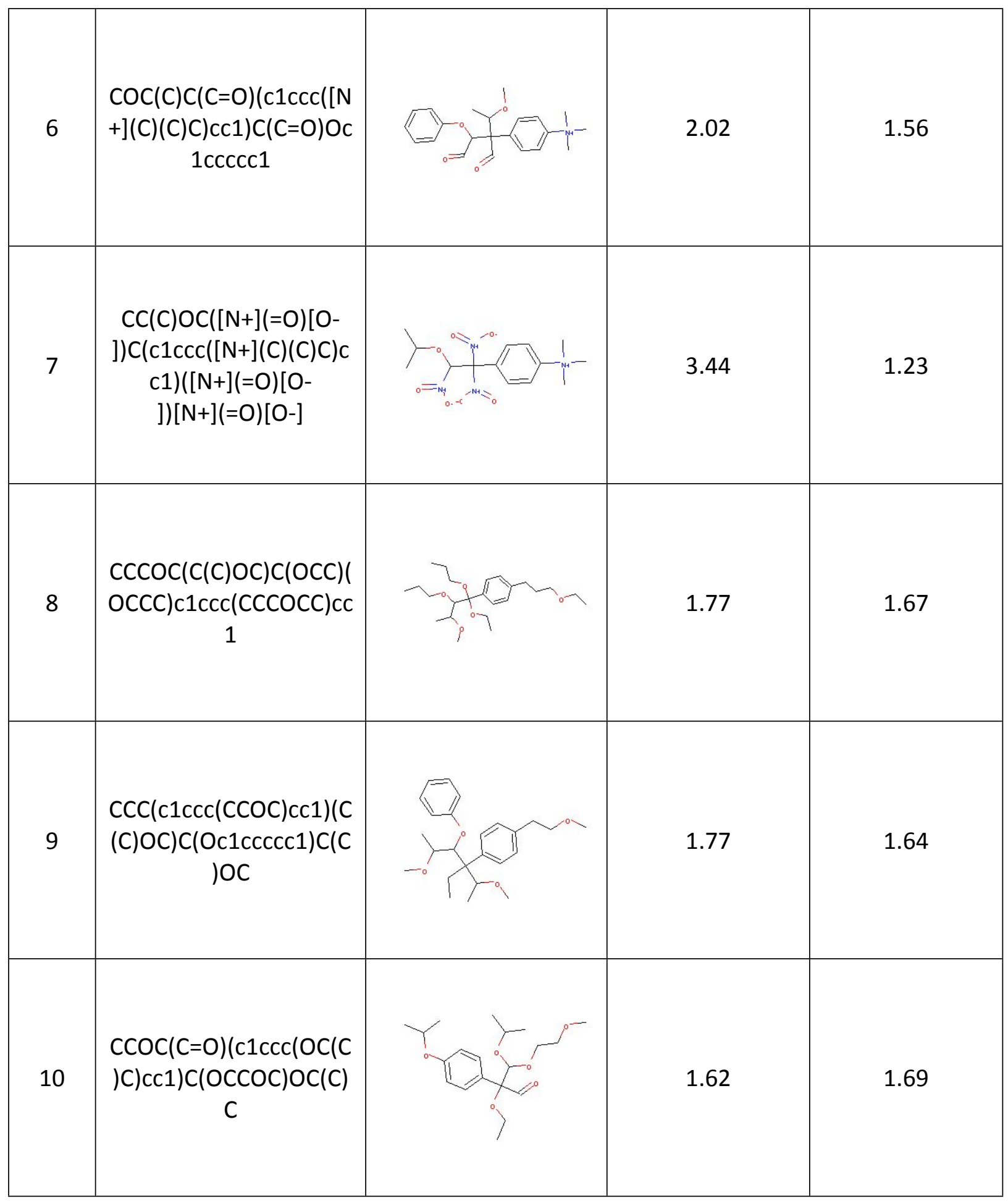




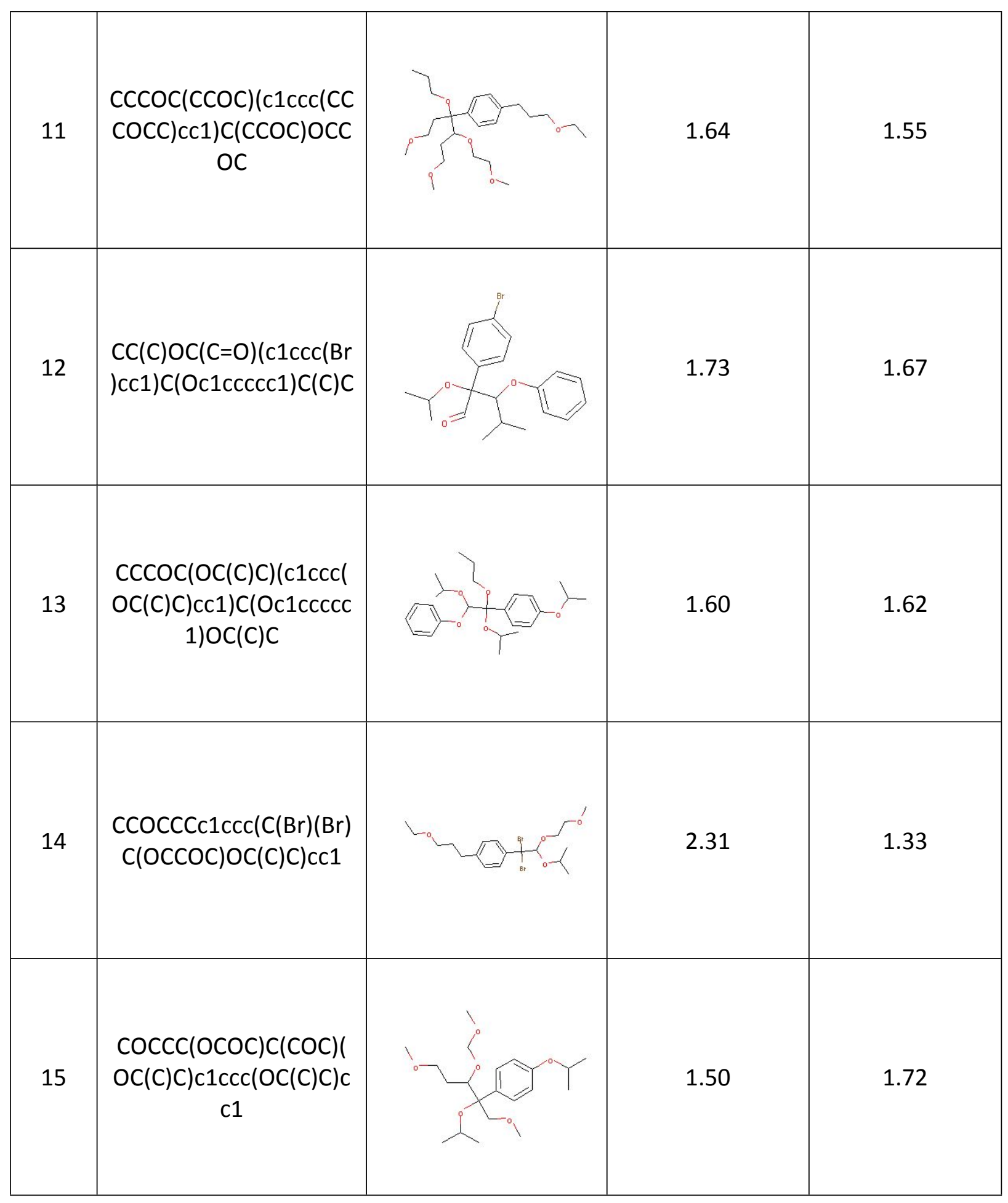




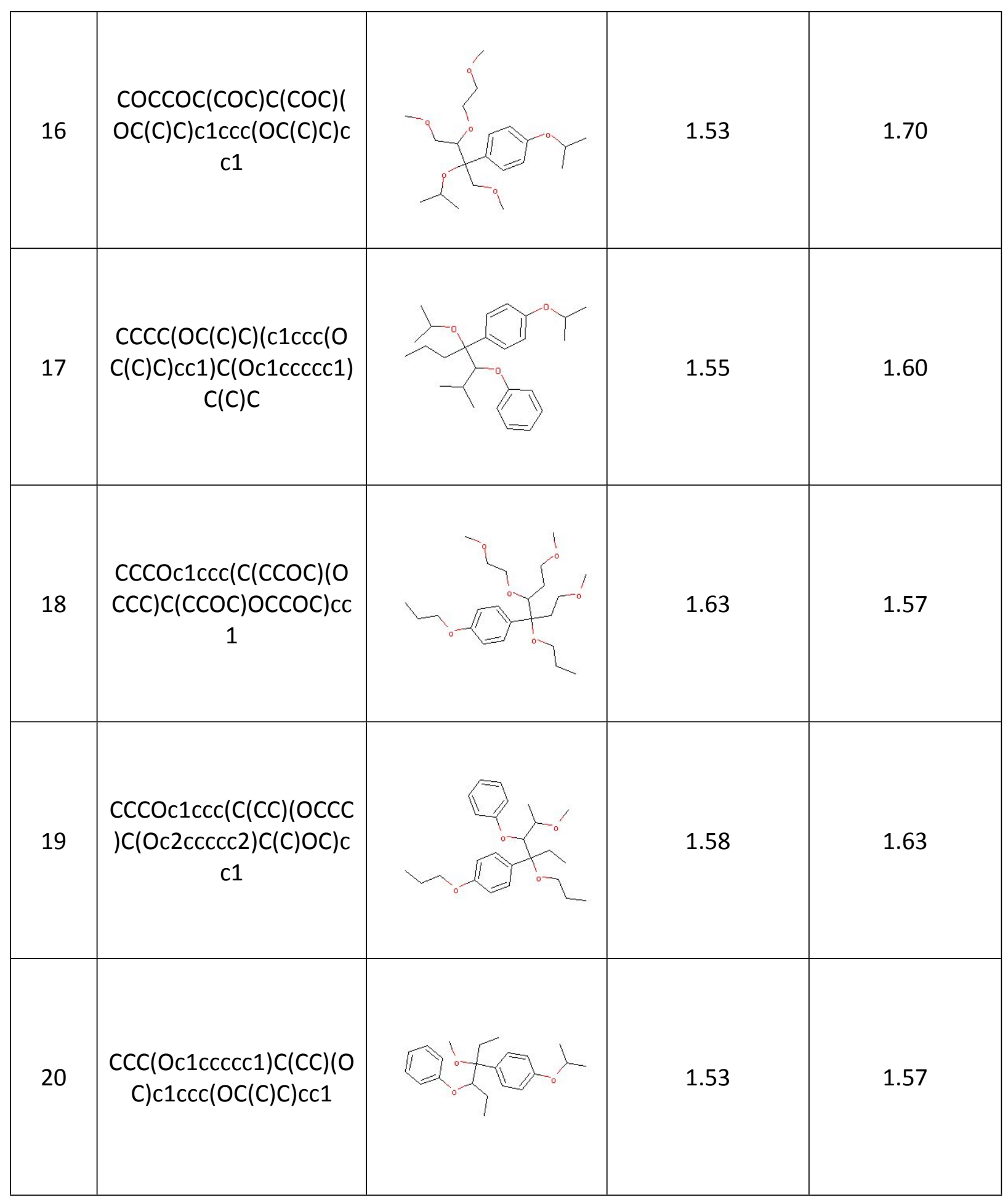




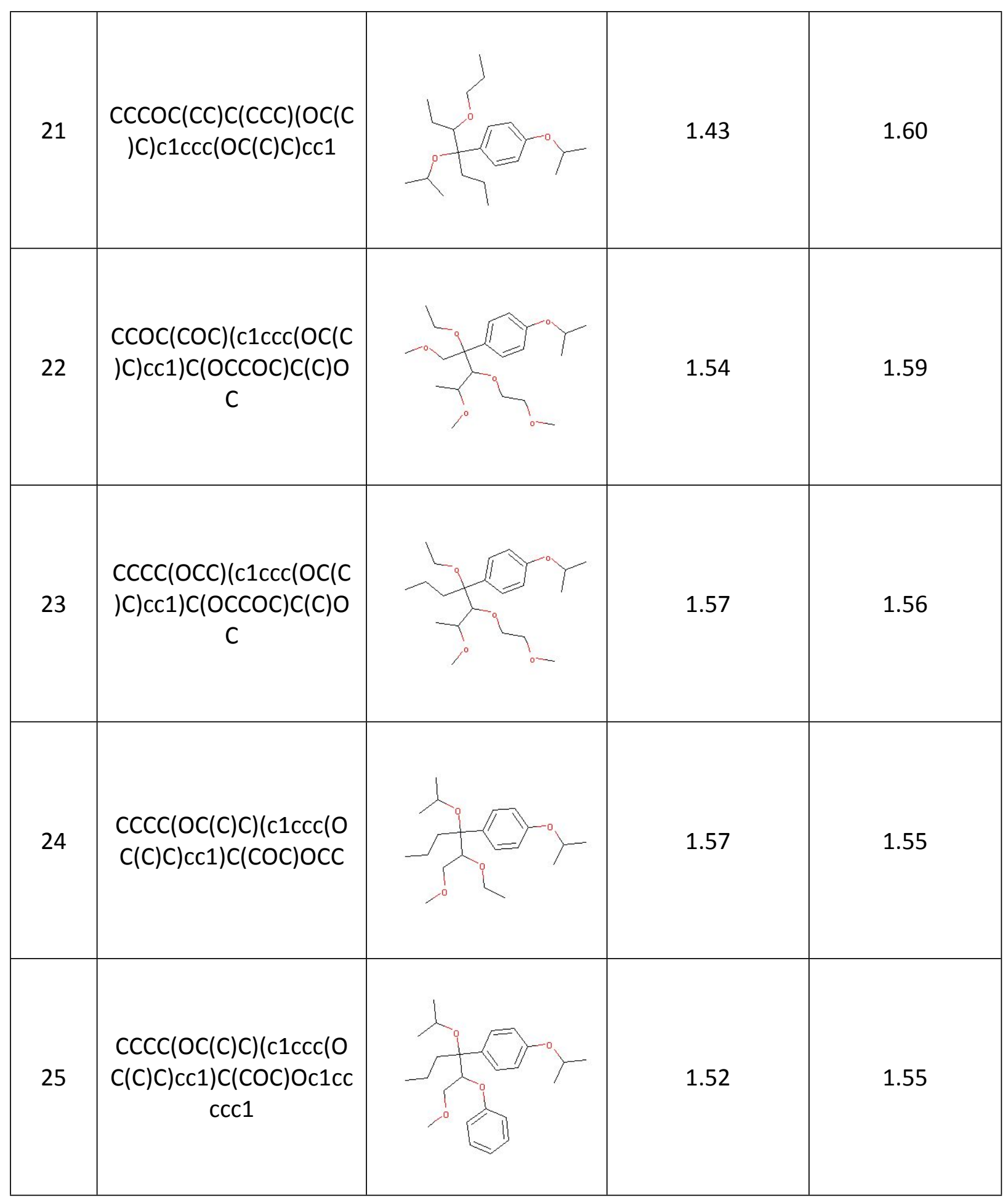




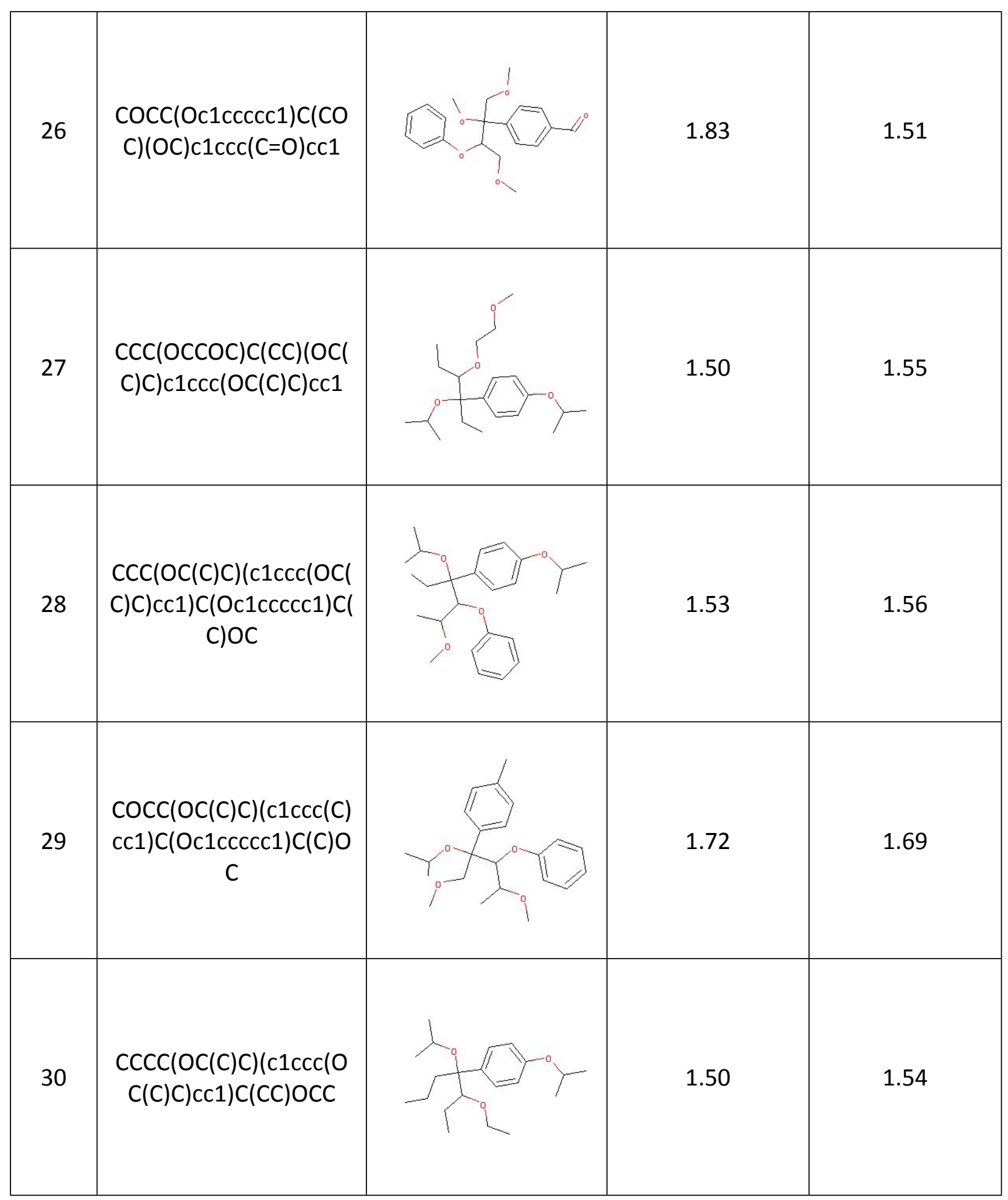




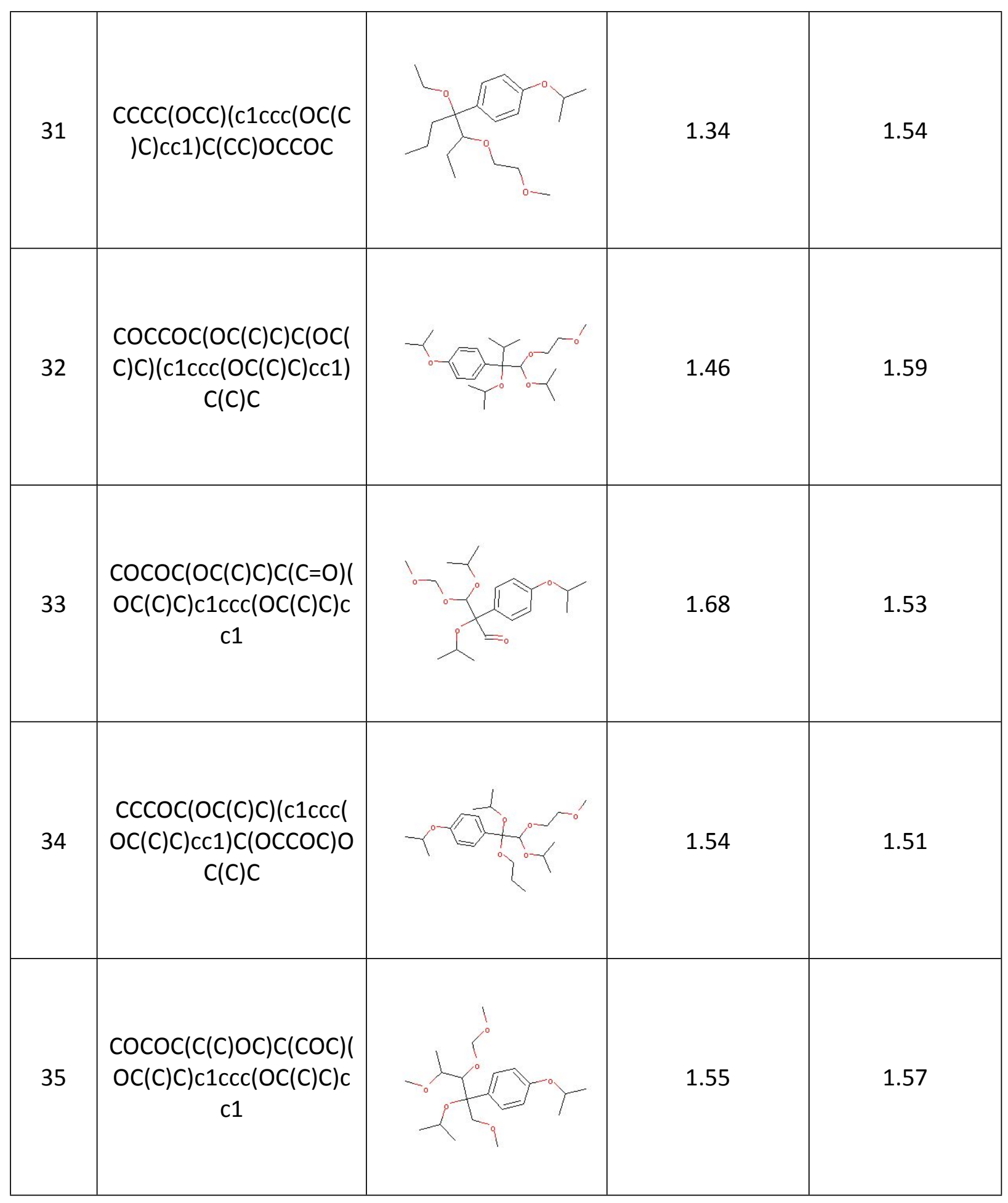




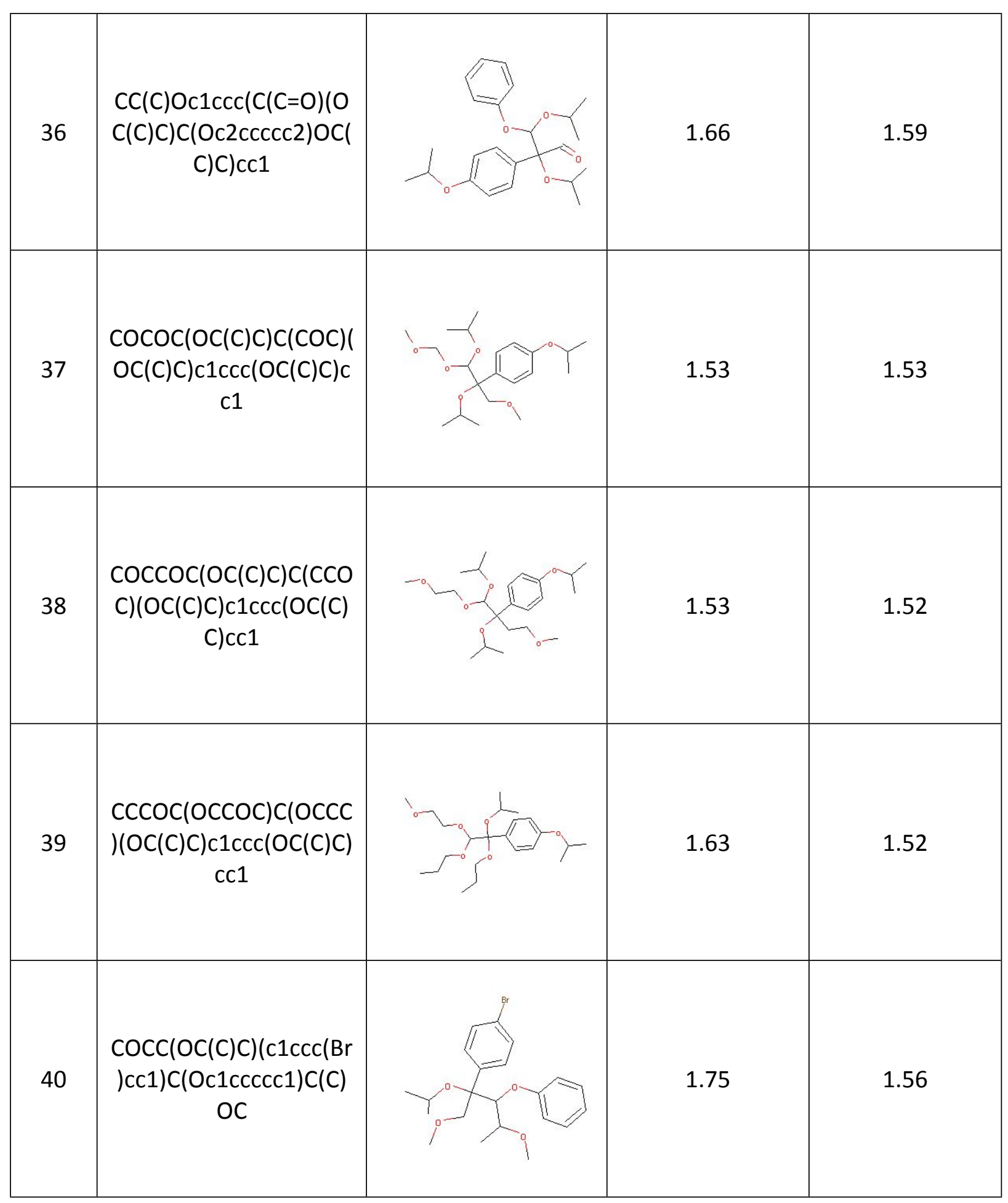


Table S6 List of HBEs evaluated during BO run \#2 (BO2) and their corresponding Eox. The initial 10 were randomly selected.

\begin{tabular}{|c|c|c|c|c|}
\hline HBE \# & SMILES & 2-D Structure & $\begin{array}{l}\text { DFT-computed } \\
\text { Ex (V vs. NHE) }^{\circ}\end{array}$ & $\begin{array}{l}\text { BO-computed } \\
\text { Ex (V vs. NHE) }^{\text {ox }}\end{array}$ \\
\hline $1^{\prime}$ & $\begin{array}{c}\operatorname{ccCOC}(\mathrm{COC})(\mathrm{c} 1 \mathrm{ccc}(\mathrm{CC} \\
\mathrm{COCC}) \operatorname{cc} 1) \mathrm{C}(\mathrm{OCC})[\mathrm{N}+]( \\
=0)\left[\mathrm{O}^{-}\right]\end{array}$ & & 2.08 & \\
\hline $2^{\prime}$ & $\begin{array}{c}\mathrm{CCCOC}(\mathrm{OCC})(\mathrm{c} 1 \mathrm{ccc}(\mathrm{CO} \\
\mathrm{C}) \mathrm{cc} 1) \mathrm{C}(\mathrm{OC})[\mathrm{N}+](=\mathrm{O})[ \\
\mathrm{O}-]\end{array}$ & & 2.29 & \\
\hline $3^{\prime}$ & $\begin{array}{c}\mathrm{CCCOc1ccc}(\mathrm{C}(\mathrm{C}(\mathrm{OCCC}) \\
\mathrm{OC}(\mathrm{C}) \mathrm{C})([\mathrm{N}+](=\mathrm{O})[\mathrm{O}- \\
])[\mathrm{N}+](=\mathrm{O})[\mathrm{O}-]) \mathrm{cc} 1\end{array}$ & & 2.07 & \\
\hline $4^{\prime}$ & $\begin{array}{c}C C C O c 1 \operatorname{ccc}(C(B r)(C=0) \\
C(C=0) O C) \operatorname{cc} 1\end{array}$ & & 1.94 & \\
\hline $5^{\prime}$ & $\begin{array}{l}C C C C(O c 1 c c c c c 1) C(C C \\
C)(\operatorname{coccc}(\operatorname{Br}) \operatorname{cc} 1) C(C) C\end{array}$ & & 1.70 & \\
\hline
\end{tabular}




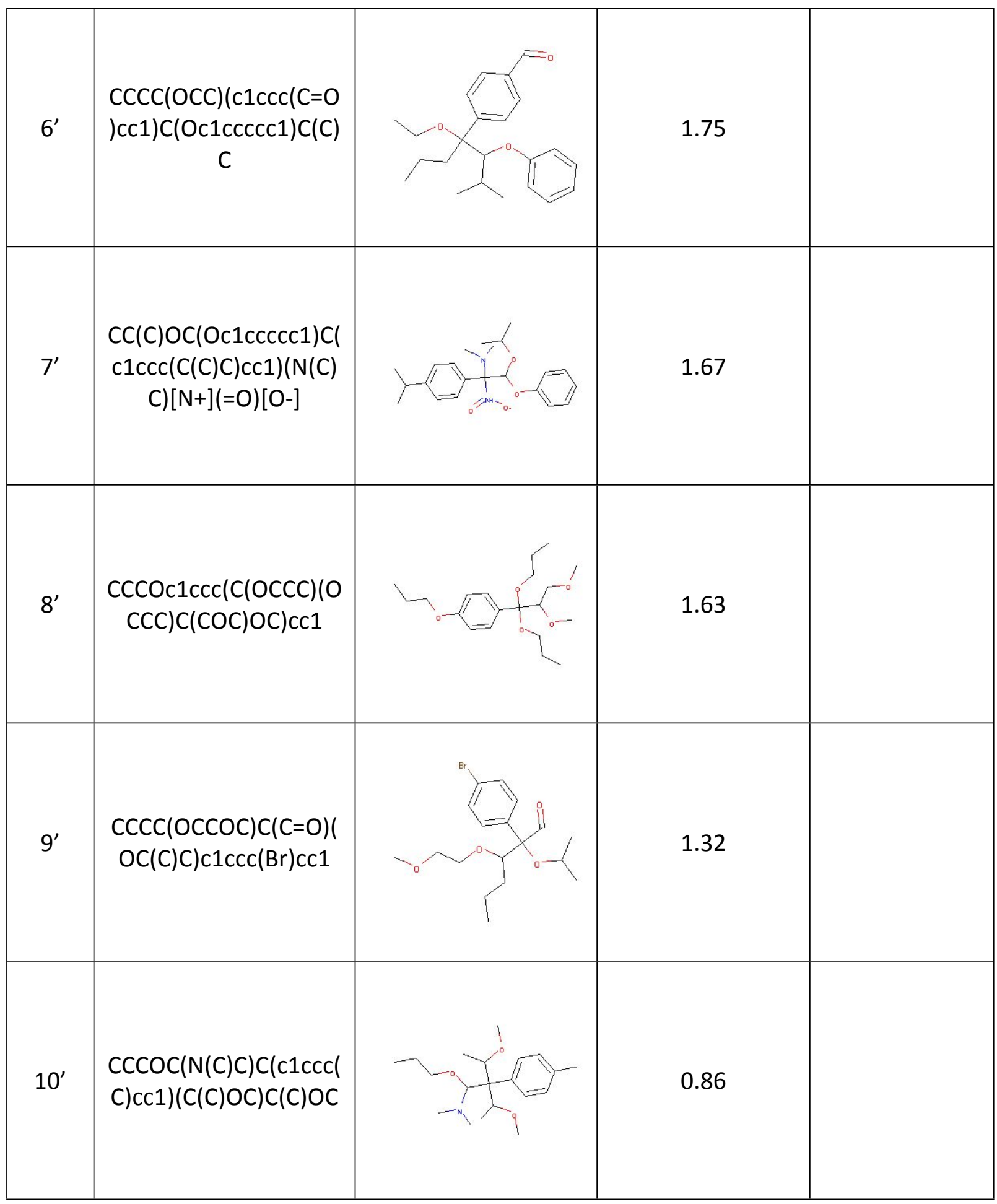




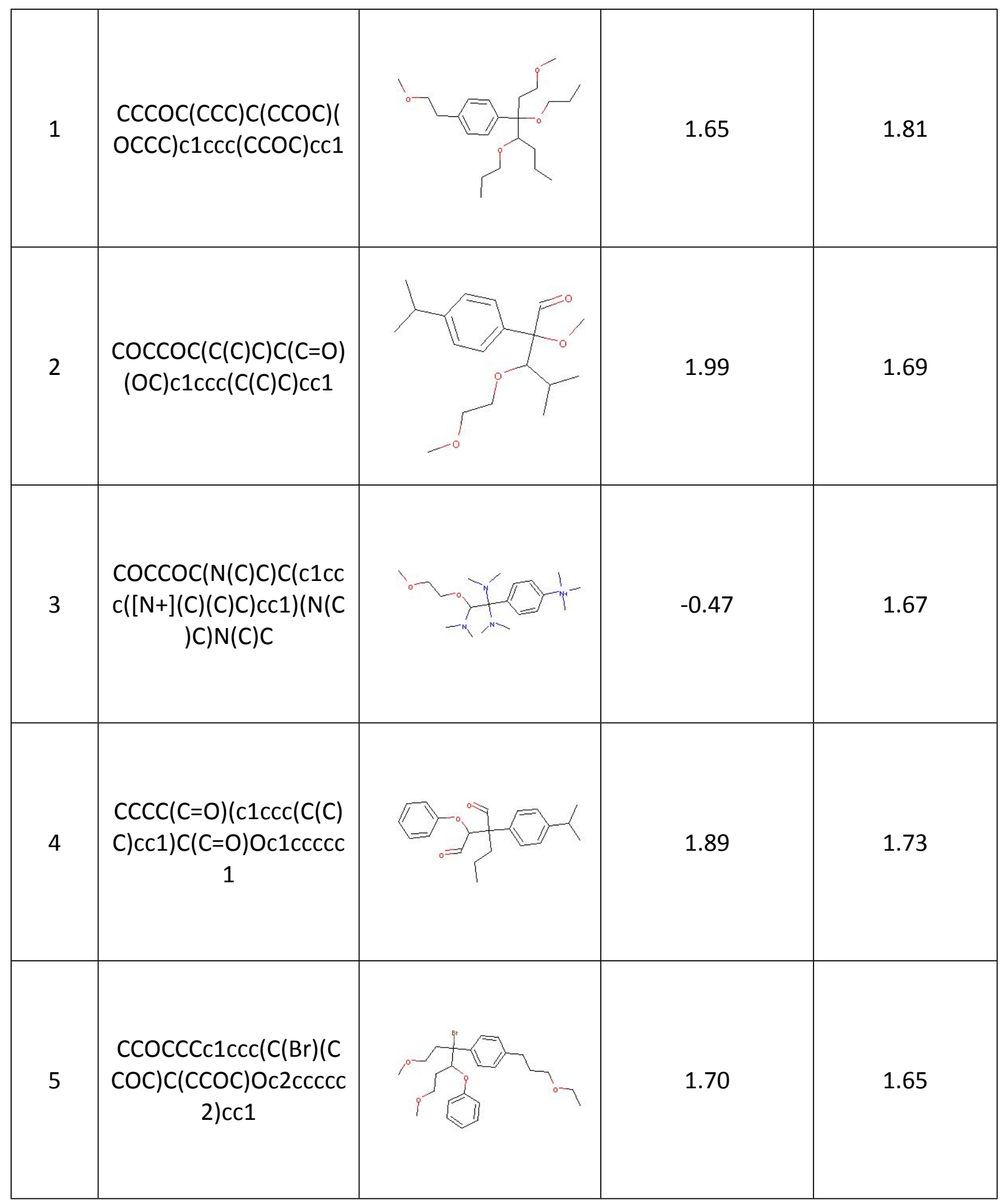




\begin{tabular}{|c|c|c|c|c|}
\hline 6 & $\begin{array}{c}\mathrm{O}=\mathrm{Cc} 1 \mathrm{ccc}(\mathrm{C}(\mathrm{C}(\mathrm{O}) 2 \mathrm{cccc} \\
\mathrm{c} 2)[\mathrm{N}+](=\mathrm{O})[\mathrm{O}- \\
])([\mathrm{N}+](=\mathrm{O})[\mathrm{O}- \\
])[\mathrm{N}+](=\mathrm{O})[\mathrm{O}-]) \mathrm{cc} 1\end{array}$ & & 2.53 & 1.68 \\
\hline 7 & $\begin{array}{c}\operatorname{cccc}(\mathrm{CCOC})(\operatorname{c1ccc}(\mathrm{C}(\mathrm{C} \\
) \mathrm{C}) \operatorname{cc} 1) \mathrm{C}(\mathrm{Oc} 1 \mathrm{ccccc} 1)[\mathrm{N} \\
+](\mathrm{C})(\mathrm{C}) \mathrm{C}\end{array}$ & & 2.29 & 1.68 \\
\hline 8 & $\begin{array}{c}\mathrm{CCCC}(\mathrm{Oc} 1 \mathrm{ccccc} 1) \mathrm{C}(\mathrm{Br}) \\
(\mathrm{Br}) \mathrm{c} 1 \mathrm{ccc}(\mathrm{C}(\mathrm{C}) \mathrm{C}) \mathrm{cc1}\end{array}$ & & 1.86 & 1.66 \\
\hline 9 & $\begin{array}{c}\operatorname{COCCOC}(\mathrm{N}(\mathrm{C}) \mathrm{C}) \mathrm{C}(\mathrm{OC})( \\
\mathrm{c} 1 \mathrm{ccc}(\mathrm{OC}(\mathrm{C}) \mathrm{C}) \mathrm{cc} 1) \mathrm{N}(\mathrm{C}) \\
\mathrm{C}\end{array}$ & & -0.33 & 1.70 \\
\hline 10 & $\begin{array}{c}\operatorname{CCCOC}(C C C) C(\operatorname{clccc}(B \\
r) \operatorname{cc} 1)(N(C) C)[N+](=0)[ \\
O-]\end{array}$ & & 1.91 & 1.56 \\
\hline
\end{tabular}




\begin{tabular}{|c|c|c|c|c|}
\hline 11 & $\begin{array}{l}C C C O c 1 \mathrm{ccc}(\mathrm{C}(\mathrm{OCCC})(0 \\
\mathrm{CCC}) \mathrm{C}(\mathrm{OCCC}) \mathrm{OCCC}) \mathrm{cc} 1\end{array}$ & & 1.68 & 1.81 \\
\hline 12 & $\begin{array}{c}\mathrm{CCCOc} 1 \mathrm{ccc}(\mathrm{C}(\mathrm{CC})(\mathrm{CCC}) \\
\mathrm{C}(\mathrm{CCC}) \mathrm{OCCC}) \mathrm{cc} 1\end{array}$ & & 1.50 & 1.90 \\
\hline 13 & $\begin{array}{c}\mathrm{CCCOc} 1 \mathrm{ccc}(\mathrm{C}(\mathrm{Br})(\mathrm{C}(\mathrm{OC} \\
\mathrm{CC}) \mathrm{OC}(\mathrm{C}) \mathrm{C})[\mathrm{N}+](\mathrm{C})(\mathrm{C}) \mathrm{C} \\
) \mathrm{cc} 1\end{array}$ & & 2.31 & 1.65 \\
\hline 14 & $\begin{array}{c}\mathrm{CCCC}(\mathrm{Oc} 1 \mathrm{ccccc} 1) \mathrm{C}(\mathrm{CC} \\
\mathrm{C})(\mathrm{OC}) \mathrm{c} 1 \mathrm{ccc}(\mathrm{Br}) \mathrm{cc} 1\end{array}$ & & 1.72 & 1.88 \\
\hline 15 & $\begin{array}{c}\mathrm{CCCOc} 1 \mathrm{ccc}(\mathrm{C}(\mathrm{CCC})(\mathrm{OC} \\
\mathrm{CC}) \mathrm{C}(\mathrm{OCCC})[\mathrm{N}+](\mathrm{C})(\mathrm{C}) \\
\mathrm{C}) \mathrm{cc} 1\end{array}$ & & 1.92 & 1.72 \\
\hline
\end{tabular}




\begin{tabular}{|c|c|c|c|c|}
\hline 16 & $\begin{array}{c}\mathrm{CCCC}(\operatorname{Oc} 1 \mathrm{ccccc} 1) \mathrm{C}(\mathrm{CC} \\
\mathrm{C})(\mathrm{c} 1 \mathrm{ccc}(\mathrm{C}(\mathrm{C}) \mathrm{OC}) \mathrm{cc} 1) \mathrm{N} \\
\text { (C)C }\end{array}$ & & 1.02 & 1.71 \\
\hline 17 & $\begin{array}{c}c \operatorname{coc} C \operatorname{cc} 1 \operatorname{ccc}(C(C=0)( \\
C=0) C(C=0) O c 2 \operatorname{ccccc} 2 \\
) \operatorname{cc} 1\end{array}$ & & 1.90 & 1.77 \\
\hline 18 & $\begin{array}{c}\mathrm{CCCOC}(\mathrm{c} 1 \mathrm{ccc}(\mathrm{CCCOCC} \\
) \mathrm{cc} 1)(\mathrm{C}(\mathrm{C}) \mathrm{OC}) \mathrm{C}(\mathrm{CCC}) \mathrm{O} \\
\mathrm{c} 1 \mathrm{ccccc} 1\end{array}$ & & 1.64 & 1.63 \\
\hline 19 & $\begin{array}{c}\operatorname{coccoc}(\mathrm{C}(\mathrm{C}) \mathrm{C}) \mathrm{C}(\mathrm{c} 1 \mathrm{cc} \\
\mathrm{c}([\mathrm{N}+](\mathrm{C})(\mathrm{C}) \mathrm{C}) \mathrm{cc} 1)(\mathrm{C}(\mathrm{C} \\
\quad \mathrm{C})[\mathrm{N}+](=\mathrm{O})[\mathrm{O}-]\end{array}$ & & 2.61 & 1.73 \\
\hline 20 & $\begin{array}{l}\operatorname{ccCOC}(\mathrm{OCCC}) \mathrm{C}(\mathrm{OCCC}) \\
(\mathrm{OC}(\mathrm{C}) \mathrm{C}) \mathrm{c} 1 \mathrm{ccc}(\mathrm{Br}) \mathrm{cc} 1\end{array}$ & & 2.26 & 1.59 \\
\hline
\end{tabular}




\begin{tabular}{|c|c|c|c|c|}
\hline 21 & $\begin{array}{c}\mathrm{ccCOC}(\mathrm{CCOC}) \mathrm{C}(\mathrm{CCOC}) \\
(\mathrm{CCOC}) \mathrm{c} 1 \mathrm{ccc}(\mathrm{C}) \mathrm{cc} 1\end{array}$ & & 1.99 & 1.75 \\
\hline 22 & $\begin{array}{c}\mathrm{CCC}(\mathrm{OCCOC}) \mathrm{C}(\mathrm{c} 1 \mathrm{ccc}(\mathrm{O} \\
\mathrm{C}(\mathrm{C}) \mathrm{C}) \mathrm{cc} 1)(\mathrm{C}(\mathrm{C}) \mathrm{OC}) \mathrm{C}(\mathrm{C} \\
) \mathrm{OC}\end{array}$ & & 1.45 & 1.63 \\
\hline 23 & $\begin{array}{l}C C C O c 1 \mathrm{ccc}(\mathrm{C}(\mathrm{CCC})(\mathrm{CC} \\
\mathrm{OC}) \mathrm{C}(\mathrm{CCC}) \mathrm{OCCC}) \mathrm{cc} 1\end{array}$ & & 1.45 & 1.62 \\
\hline 24 & $\begin{array}{c}\mathrm{CCCOC}(\mathrm{OCC}) \mathrm{C}(\mathrm{OCCC})( \\
\mathrm{c} 1 \mathrm{ccc}(\mathrm{C}) \mathrm{cc} 1)[\mathrm{N}+](\mathrm{C})(\mathrm{C}) \\
\mathrm{C}\end{array}$ & & 2.53 & 1.63 \\
\hline 25 & $\begin{array}{c}\mathrm{CCOCCCc1} 1 \mathrm{ccc}(\mathrm{C}(\mathrm{OC})(\mathrm{O} \\
\mathrm{C}) \mathrm{C}(\mathrm{C}=0) \mathrm{Oc} 2 \mathrm{ccccc} 2) \mathrm{cc} \\
1\end{array}$ & & 1.89 & 1.65 \\
\hline
\end{tabular}




\begin{tabular}{|c|c|c|c|c|}
\hline 26 & $\begin{array}{c}\mathrm{CCOCCCc1} \operatorname{ccc}(\mathrm{C}(\mathrm{C}=\mathrm{O})( \\
\mathrm{C}(\mathrm{C}) \mathrm{OC}) \mathrm{C}(\mathrm{CCOC}) \mathrm{OCCO} \\
\mathrm{C}) \mathrm{cc} 1\end{array}$ & & 1.99 & 1.63 \\
\hline 27 & $\begin{array}{c}\mathrm{CCCOC}(\mathrm{C}(\mathrm{C}) \mathrm{OC}) \mathrm{C}(\mathrm{C}=\mathrm{O}) \\
(\mathrm{c} 1 \mathrm{ccc}(\mathrm{CCOC}) \mathrm{cc} 1) \mathrm{C}(\mathrm{C}) \\
\mathrm{OC}\end{array}$ & & 2.01 & 1.73 \\
\hline 28 & $\begin{array}{c}\mathrm{cCCC}(\operatorname{Oc} 1 \mathrm{ccccc} 1) \mathrm{C}(\mathrm{CC} \\
\mathrm{C})(\mathrm{CCOC}) \mathrm{c} 1 \mathrm{ccc}(\mathrm{CCOC}) \mathrm{c} \\
\mathrm{c1}\end{array}$ & & 1.67 & 1.68 \\
\hline 29 & $\begin{array}{c}\mathrm{CCCC}(\mathrm{CC})(\mathrm{c} 1 \mathrm{ccc}(\mathrm{CCCO} \\
\mathrm{CC}) \mathrm{cc} 1) \mathrm{C}(\mathrm{CC}) \operatorname{Oc} 1 \mathrm{ccccc} \\
1\end{array}$ & & 1.69 & 1.86 \\
\hline 30 & $\begin{array}{c}\operatorname{cccOC}(\mathrm{OCCC})(\mathrm{c} 1 \mathrm{ccc}(\mathrm{B} \\
\mathrm{r}) \mathrm{cc} 1) \mathrm{C}(\mathrm{Oc} 1 \mathrm{ccccc} 1)[\mathrm{N}+ \\
](=0)[0-]\end{array}$ & & 2.01 & 1.60 \\
\hline
\end{tabular}




\begin{tabular}{|c|c|c|c|c|}
\hline 31 & $\begin{array}{c}\mathrm{CCCOc} 1 \mathrm{ccc}(\mathrm{C}(\mathrm{CCC})(\mathrm{CC} \\
\mathrm{C}) \mathrm{C}(\mathrm{OC}) \mathrm{C}(\mathrm{C}) \mathrm{C}) \mathrm{cc} 1\end{array}$ & & 1.47 & 1.70 \\
\hline 32 & $\begin{array}{c}\mathrm{CCCO} 1 \mathrm{ccc}(\mathrm{C}(\mathrm{CCC})(\mathrm{CC} \\
\mathrm{C}) \mathrm{C}(\mathrm{Oc} 2 \mathrm{ccccc} 2)[\mathrm{N}+](= \\
0)[\mathrm{O}-]) \mathrm{cc} 1\end{array}$ & & 1.72 & 1.81 \\
\hline 33 & $\begin{array}{c}\mathrm{ccCOC}(\mathrm{OCCC})(\mathrm{c} 1 \mathrm{ccc}(\mathrm{C} \\
\mathrm{ccOCC}) \operatorname{cc} 1) \mathrm{C}(\mathrm{CCC}) \mathrm{Oc} 1 \\
\operatorname{ccccc} 1\end{array}$ & & 1.72 & 1.56 \\
\hline 34 & $\begin{array}{c}\mathrm{CCCO} 1 \mathrm{ccc}(\mathrm{C}(\mathrm{CCC})(\mathrm{OC} \\
(\mathrm{C}) \mathrm{C}) \mathrm{C}(\mathrm{CCC}) \mathrm{OCCOC}) \mathrm{cc} \\
1\end{array}$ & & 1.64 & 1.65 \\
\hline 35 & $\begin{array}{c}\mathrm{CCCOc} 1 \mathrm{ccc}(\mathrm{C}(\mathrm{CC})(\mathrm{CCO} \\
\mathrm{C}) \mathrm{C}(\mathrm{CCOC}) \mathrm{OC}) \mathrm{cc} 1\end{array}$ & & 1.52 & 1.70 \\
\hline
\end{tabular}




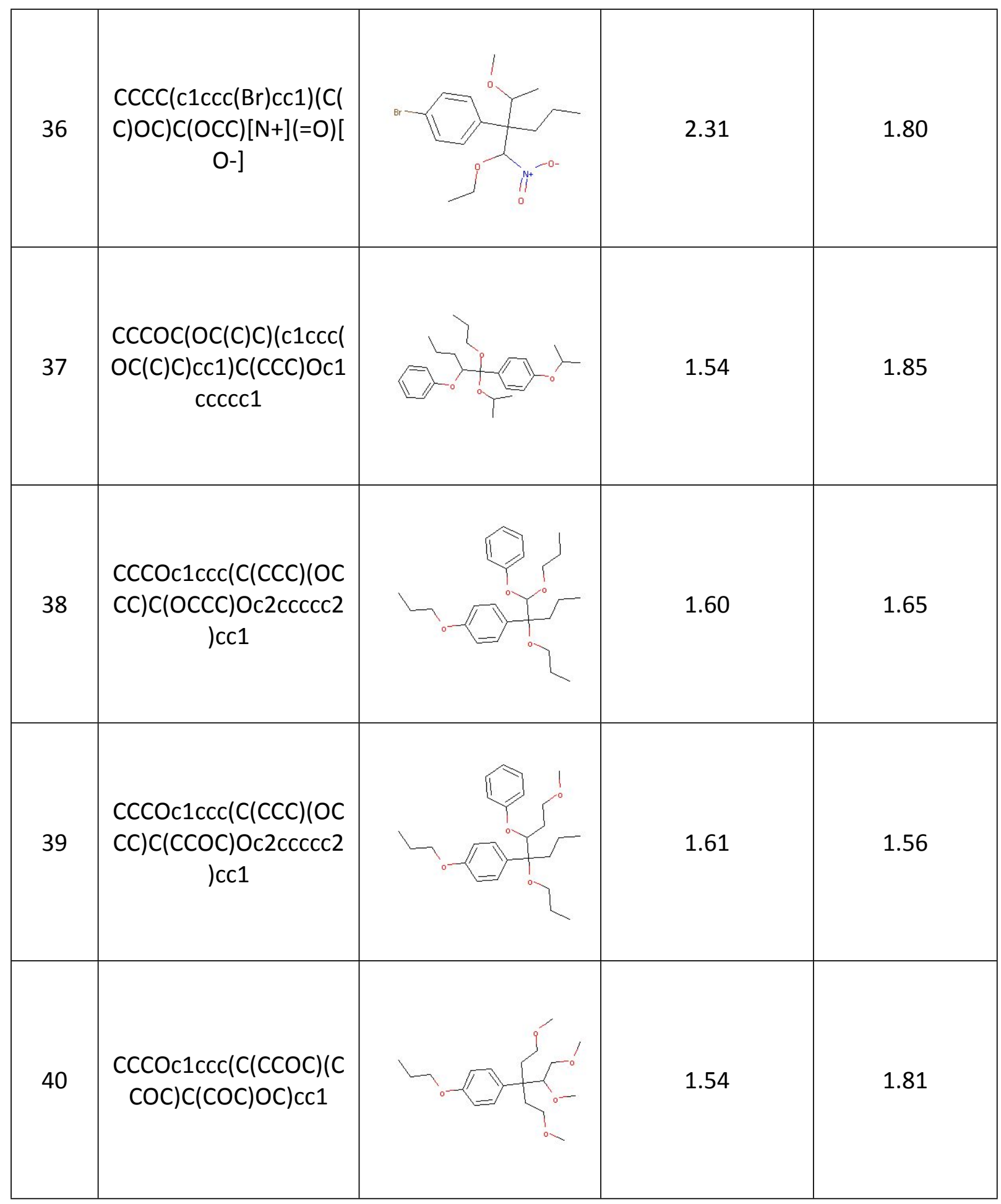




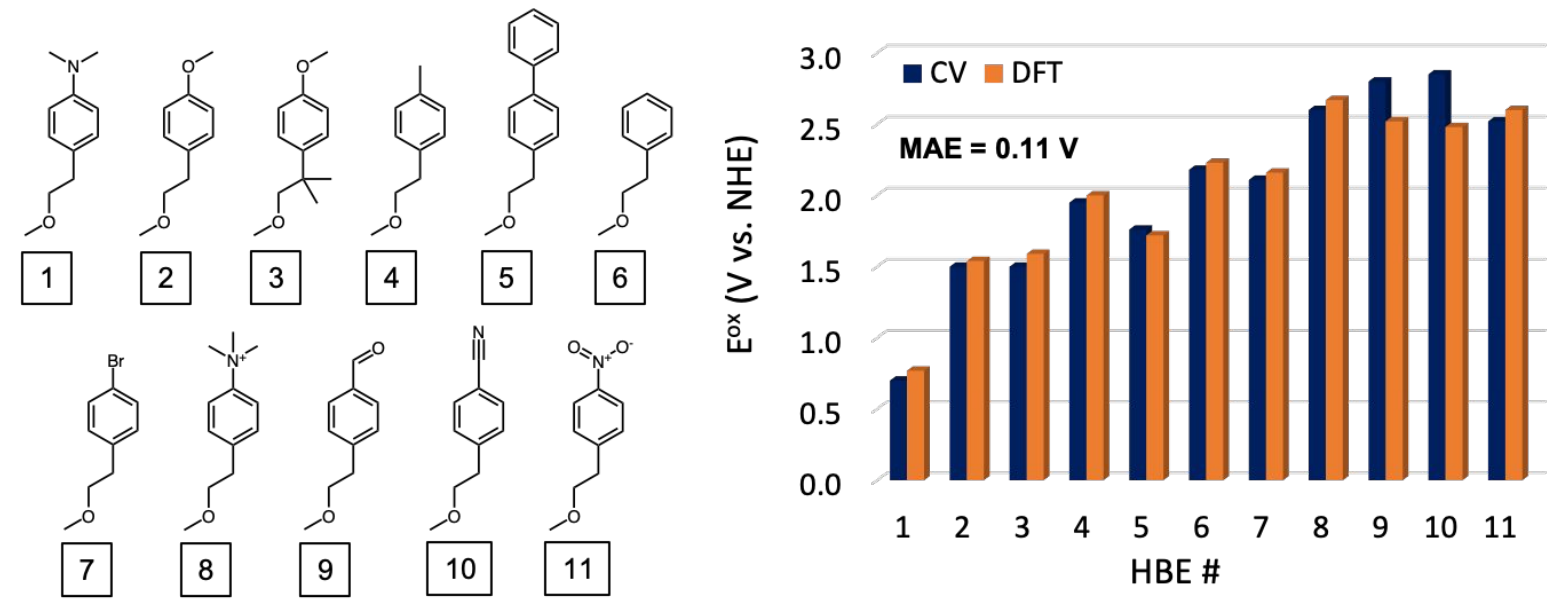

Figure S1 Comparison between DFT-computed oxidation potentials (orange) and cyclic voltammetry measurements (blue) for 11 randomly selected homobenzylic ether molecules
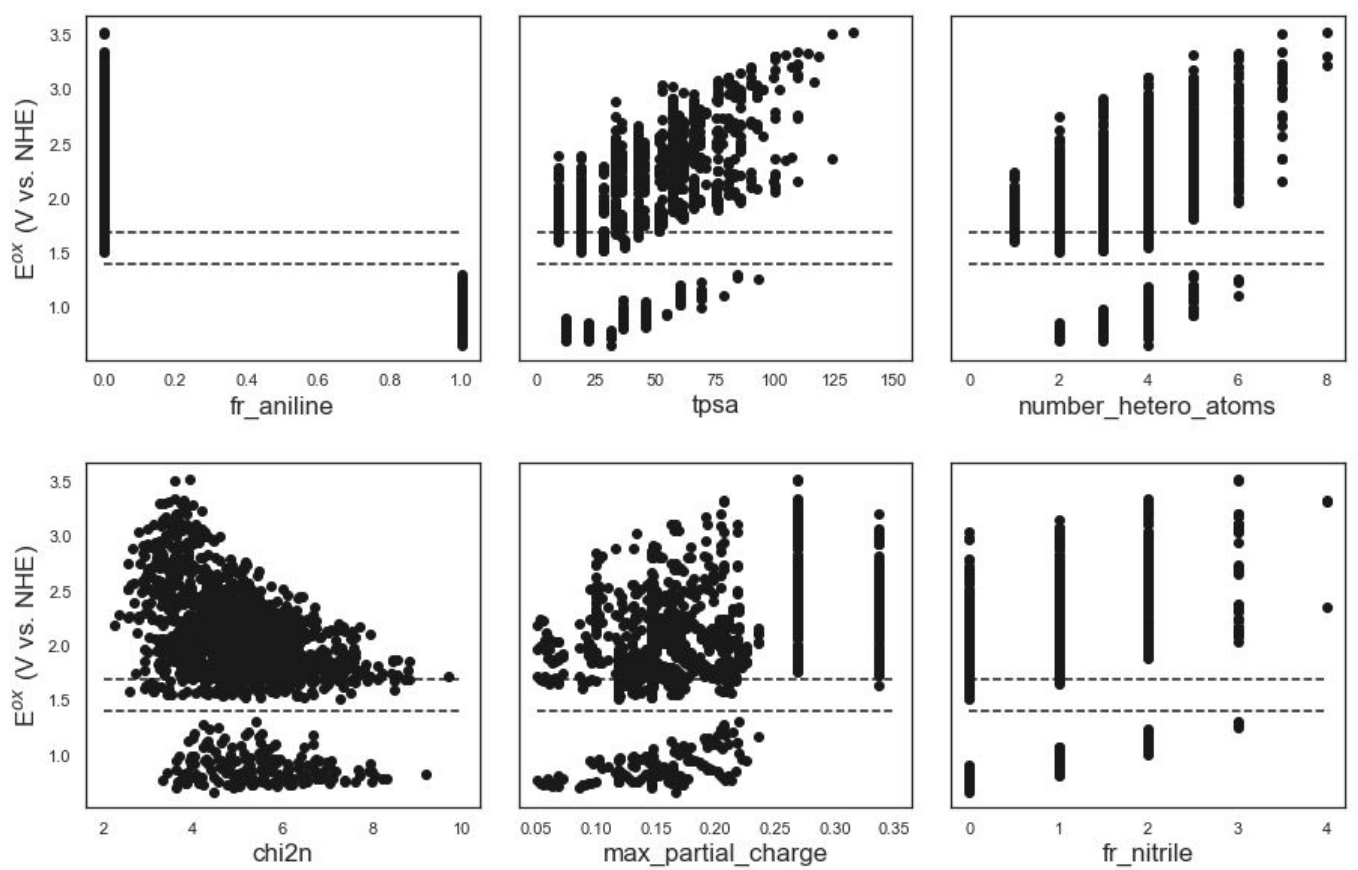

Figure S2 DFT-computed Ex as a function of 6 different features with highest Pearson correlation coefficient values. 


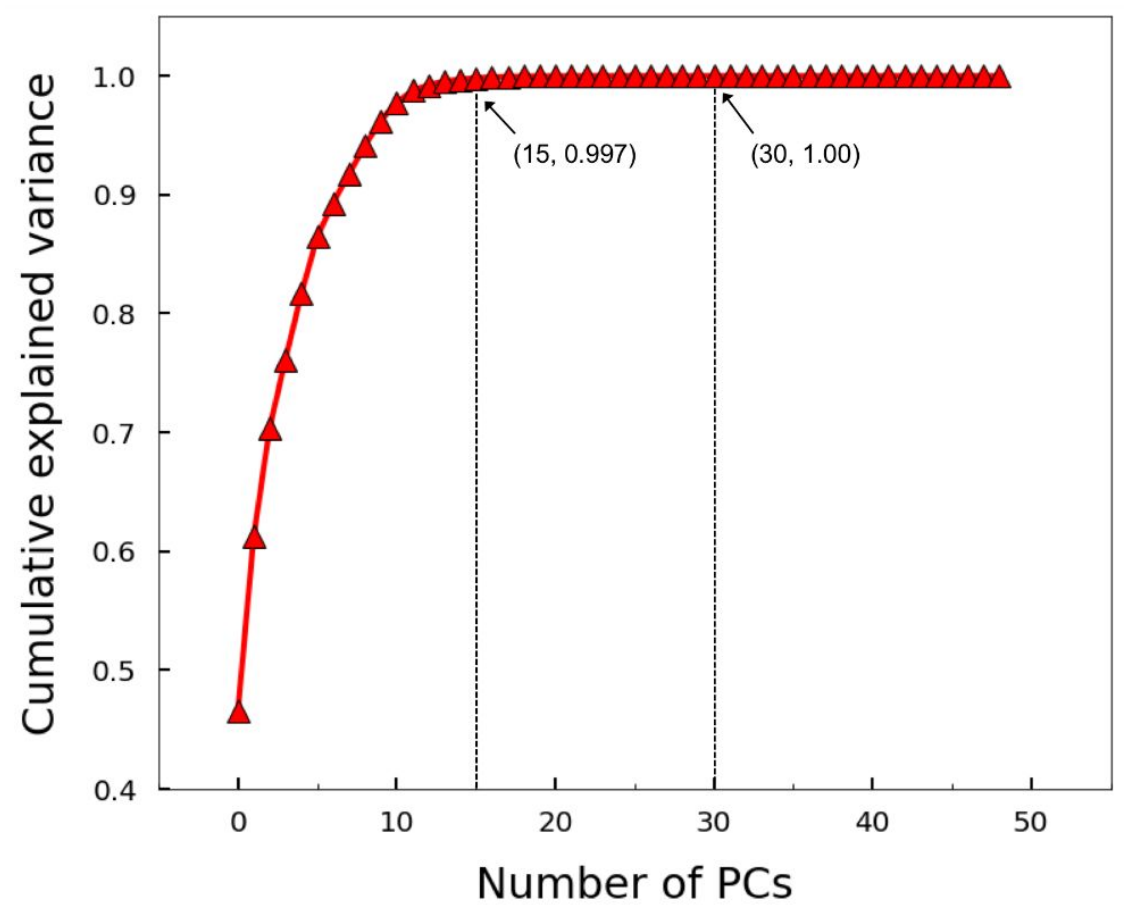

Figure S3 PCA analysis of 49 generated features for 1,400 HBE data set

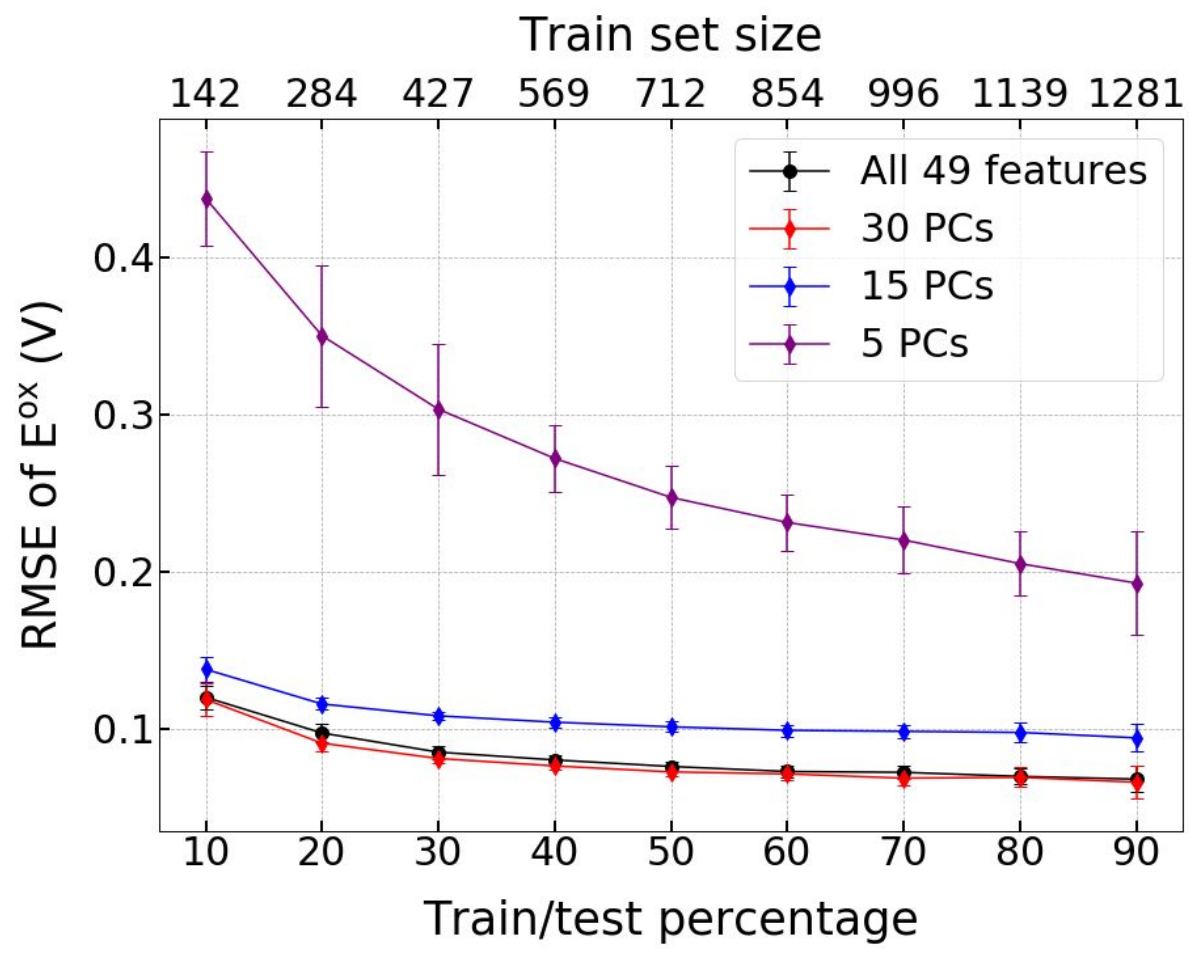

Figure S4 Learning curves of Gaussian Process Regression using various feature sets 


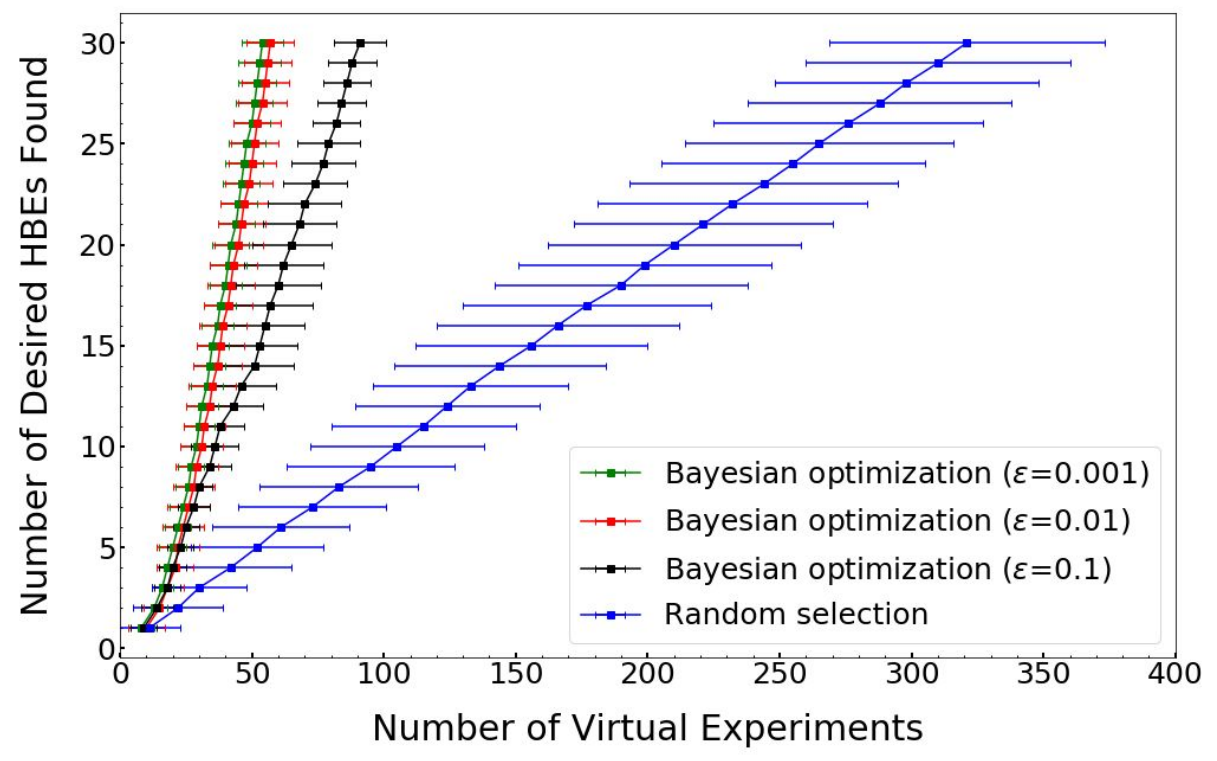

Figure S5 Comparison of sampling efficiency between random selection and Bayesian optimizations with different values of epsilon

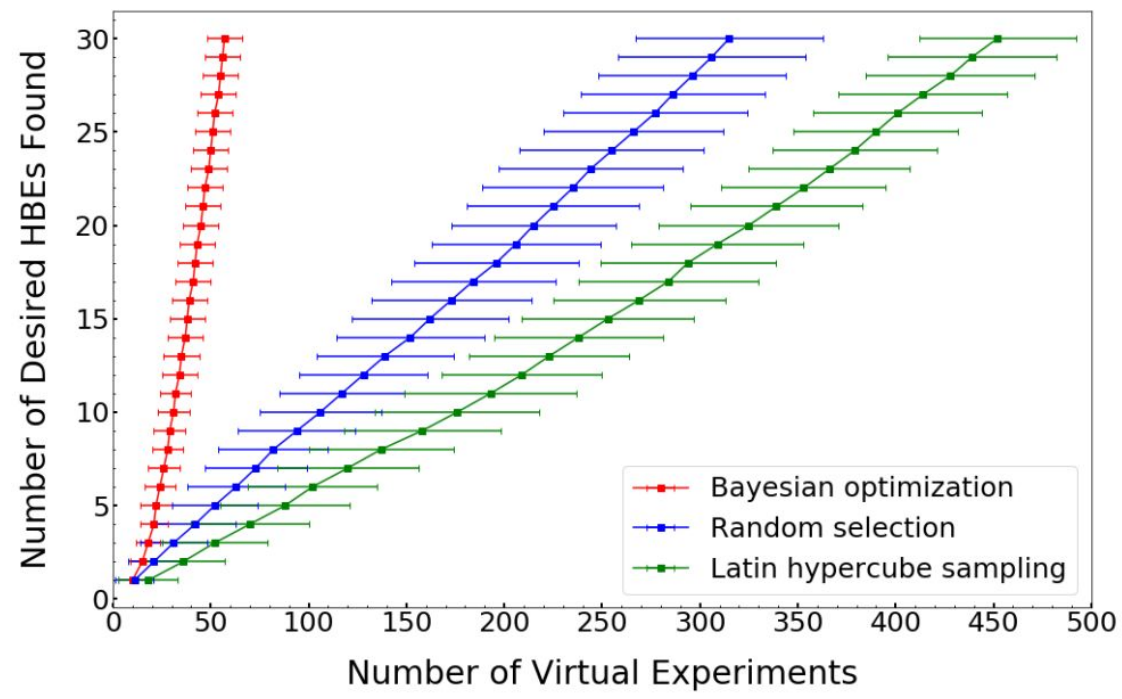

Figure S6 Comparison of efficiency between Bayesian optimization, random selection and Latin hypercube sampling

\section{References}

1. Wildman, S. A.; Crippen, G. M., Prediction of Physicochemical Parameters by Atomic Contributions. Journal of Chemical Information and Computer Sciences 1999, 39, (5), 868-873.

2. $\quad$ Ertl, P.; Rohde, B.; Selzer, P., Fast Calculation of Molecular Polar Surface Area as a Sum of Fragment-Based Contributions and Its Application to the Prediction of Drug Transport Properties. Journal of Medicinal Chemistry 2000, 43, (20), 3714-3717.

3. Hall, L. H.; Kier, L. B., The Molecular Connectivity Chi Indexes and Kappa Shape Indexes in Structure-Property Modeling. In Reviews in Computational Chemistry, 2007; pp 367-422.

4. Labute, P., A widely applicable set of descriptors. Journal of Molecular Graphics and Modelling 2000, 18, (4), $464-477$. 